\title{
Topological partial *-algebras: Basic properties and examples
}

\author{
J.-P. Antoine ${ }^{1}$, F. Bagarello ${ }^{2}$ and C. Trapani ${ }^{3}$ \\ ${ }^{1}$ Institut de Physique Théorique, Université Catholique de Louvain \\ B-1348 Louvain-la-Neuve, Belgium \\ 2 Dipartimento di Matematica dell' Università di Palermo \\ I-90123 Palermo, Italy \\ ${ }^{3}$ Istituto di Fisica dell' Università di Palermo \\ I-90123 Palermo, Italy
}

\begin{abstract}
Let $\mathfrak{A}$ be a partial *-algebra endowed with a topology $\tau$ that makes it into a locally convex topological vector space $\mathfrak{A}[\tau]$. Then $\mathfrak{A}$ is called a topological partial *-algebra if it satisfies a number of conditions, which all amount to require that the topology $\tau$ fits with the multiplier structure of $\mathfrak{A}$. Besides the obvious cases of topological quasi *-algebras and $\mathrm{CQ}^{*}$-algebras, we examine several classes of potential topological partial *-algebras, either function spaces (lattices of $L^{p}$ spaces on $[0,1]$ or on $\mathbb{R}$, amalgam spaces), or partial *-algebras of operators (operators on a partial inner product space, $\mathrm{O}^{*}$-algebras).
\end{abstract}

E-mail: Antoine@fyma.ucl.ac.be

Bagarello@ipamat.math.unipa.it

Trapani@mbox.unipa.it

UCL-IPT-97-16

November 1997 


\section{Introduction and motivation}

A partial *-algebra is a vector space equipped with a multiplication that is only defined for certain pairs of elements. Many different species have cropped up in the recent mathematical literature, for instance, quasi *-algebras [29, 30, $\mathrm{CQ}^{*}$-algebras [15, 16] or various kinds of partial *-algebras of operators in Hilbert spaces, the so-called partial $\mathrm{O}^{*}$-algebras [6]-12. In all cases, there is an algebraic backbone, the abstract partial *-algebra, mentioned in [23] and developed in [6] and [9]. On top of that, a number of topological properties are introduced. For instance, partial $\mathrm{O}^{*}$-algebras were envisaged as generalizations of *-algebras of bounded operators (von Neumann algebras or $\mathrm{C}^{*}$-algebras) and of *-algebras of unbounded operators or $\mathrm{O}^{*}$-algebras [34].

Yet one element is missing in this picture, an abstract notion of topological partial *-algebra, that would encompass and unify all these examples. That such a concept is useful is illustrated by the following situation.

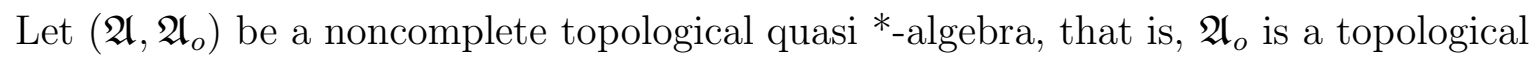
*-algebra, but the multiplication is only separately, not jointly continuous for the topology of $\mathfrak{A}_{o}$, and the latter is not complete. If $\mathfrak{A}$ is the completion of $\mathfrak{A}_{o}$, then it is no longer an algebra in general, but only a partial algebra: a product $A B$ is defined only (by continuity) if either $A$ or $B$ belongs to $\mathfrak{A}_{o}$. Let now $\pi_{o}$ be a ${ }^{*}$-representation of $\mathfrak{A}_{o}$ by operators acting on a dense domain $\mathcal{D}\left(\pi_{o}\right)$ in a Hilbert space $\mathcal{H}$. This means, in particular, that $\pi_{o}$ maps $\mathfrak{A}_{o}$ into $\mathcal{L}^{\dagger}\left(\mathcal{D}\left(\pi_{o}\right)\right)$, i.e. the space of all closable operators $A$ in $\mathcal{H}$ with domain $D(A)=\mathcal{D}\left(\pi_{o}\right)$ and leaving it invariant. Now it is legitimate to ask how one could extend the representation $\pi_{o}$ from $\mathfrak{A}_{o}$ to $\mathfrak{A}$, or at least to some larger subset of it. The obvious way would be by taking limits, using some notion of closability of the representation $\pi_{o}$. But this implies in general extending $\pi_{o}$ beyond $\mathcal{D}\left(\pi_{o}\right)$, since the extended operators need no longer map $\mathcal{D}\left(\pi_{o}\right)$ into itself. In [14] we have perfromed such an extension, by operators in $\mathcal{L}\left(\mathcal{D}\left(\pi_{o}\right), \mathcal{D}^{\prime}\right)$, where $\mathcal{D}^{\prime}$ is the dual of $\mathcal{D}\left(\pi_{o}\right)$ in a suitable topology. However, from the point of view of partial $\mathrm{O}^{*}$-algebras, a more natural framework for the extension is the space $\mathcal{L}^{\dagger}\left(\mathcal{D}\left(\pi_{o}\right), \mathcal{H}\right)$ of all closable operators $A$ in $\mathcal{H}$ such that $D(A)=\mathcal{D}$ and $D\left(A^{*}\right) \supset \mathcal{D}$, which is a partial *-algebra. However, in order to perform such an extension by closure, one clearly needs a more sophisticated topological structure on $\mathcal{L}^{\dagger}\left(\mathcal{D}\left(\pi_{o}\right), \mathcal{H}\right)$ than the one available in the current literature. As a matter of fact, a large number of interesting results have been obtained concerning partial O*-algebras, such as structure results, (GNS) representations, automorphisms and derivations (see [12] for a review and references to the original papers), but the interplay between the (partial) algebraic structure and the topological properties of partial $\mathrm{O}^{*}$-algebras has been largely ignored.

It is the aim of the present paper to try and fill this gap. In other words, we want 
to find a working definition of topological partial *-algebra that would cover all the cases mentioned at the beginning. Actually these fall into three categories.

(i) Simple cases, such as quasi *-algebras and $\mathrm{CQ}^{*}$-algebras, whose structure of partial *-algebra is almost trivial — but, of course, they have a rich topological structure.

(ii) Partial *-algebras of functions, such as the scale of the $L^{p}$ spaces on $[0,1]$ or the lattice generated by the family $\left\{L^{p}(\mathbb{R}), 1 \leqslant p \leqslant \infty\right\}$. These partial *-algebras have the peculiarity of carrying two structures: they are simultaneously a partial inner product space (PIP-space) [1]-3] and an abelian partial *-algebra under pointwise multiplication - and the two structures fit perfectly. We will say more about this class in Section 4 below.

(iii) Partial *-algebras of operators, such as sets of operators on a PIP-space or partial $\mathrm{O}^{*}$-algebras. Here the algebraic structure is richer and we will have only partial results (see Section 5).

This discussion at the same time suggests the organization of the paper. First we start from an abstract partial *-algebra, focusing on the structure of its multiplier spaces, as described in [4], this is Section 2. In Section 3 we propose a definition of topological partial *-algebra, based on the multiplier structure (which embodies all the information about the partial multiplication), and check that it applies indeed to the simple cases mentioned under (i) above. Sections 4 and 5 contain a full discussion of the cases (ii) and (iii), respectively. In addition to the $L^{p}$ spaces, we will also consider in Section 4 a wide class of generalizations, the so-called amalgam spaces introduced by N. Wiener [38].

This paper by no means pretends to exhaust the subject. On the contrary, it is more a program, with many questions remaining open. Yet we feel the proposed definition is natural, in the sense that, in the examples mentioned, it brings almost perfect coincidence between the algebraic (multiplier) structure and the topological one. Only applications will tell whether our definition has to be made more (or less) restrictive.

\section{Spaces of multipliers on partial *-algebras}

For the sake of completeness, we recall first the basic definitions. A partial *-algebra is a complex vector space $\mathfrak{A}$, endowed with an involution $x \mapsto x^{*}$ (that is, a bijection such that $x^{* *}=x$ ) and a partial multiplication defined by a set $\Gamma \subset \mathfrak{A} \times \mathfrak{A}$ (a binary relation) such that:

(i) $(x, y) \in \Gamma$ implies $\left(y^{*}, x^{*}\right) \in \Gamma$;

(ii) $\left(x, y_{1}\right),\left(x, y_{2}\right) \in \Gamma$ implies $\left(x, \lambda y_{1}+\mu y_{2}\right) \in \Gamma, \forall \lambda, \mu \in \mathbb{C}$; 
(iii) for any $(x, y) \in \Gamma$, there is defined a product $x \cdot y \in \mathfrak{A}$, which is distributive w.r. to the addition.

Notice that the partial multiplication is not required to be associative (and often it is not). We shall assume the partial *-algebra $\mathfrak{A}$ contains a unit $e$, i.e. $e^{*}=e,(e, x) \in \Gamma, \forall x \in \mathfrak{A}$, and $e \cdot x=x \cdot e=x, \forall x \in \mathfrak{A}$. (If $\mathfrak{A}$ has no unit, it may always be embedded into a larger partial *-algebra with unit, in the standard fashion [7].)

Given the defining set $\Gamma$, spaces of multipliers are defined in the obvious way:

$$
\begin{aligned}
(x, y) \in \Gamma & \Longleftrightarrow x \in L(y) \text { or } x \text { is a left multiplier of } y \\
& \Longleftrightarrow y \in R(x) \text { or } y \text { is a right multiplier of } x .
\end{aligned}
$$

For any subset $\mathfrak{N} \subset \mathfrak{A}$, we write

$$
L \mathfrak{N}=\bigcap_{x \in \mathfrak{N}} L(x), \quad R \mathfrak{N}=\bigcap_{x \in \mathfrak{N}} R(x)
$$

and, of course, the involution exchanges the two:

$$
(L \mathfrak{N})^{*}=R \mathfrak{N}^{*}, \quad(R \mathfrak{N})^{*}=L \mathfrak{N}^{*} .
$$

Clearly all these multiplier spaces are vector subspaces of $\mathfrak{A}$, containing $e$.

The partial *-algebra is abelian if $L(x)=R(x), \forall x \in \mathfrak{A}$, and then $x \cdot y=y \cdot x, \forall x \in$ $L(y)$. In that case, we write simply for the multiplier spaces $L(x)=R(x) \equiv M(x), L \mathfrak{N}=$ $R \mathfrak{N} \equiv M \mathfrak{N}(\mathfrak{N} \subset \mathfrak{A})$.

Now the crucial fact is that the couple of maps $(L, R)$ defines a Galois connection on the complete lattice of all vector subspaces of $\mathfrak{A}$ (ordered by inclusion), which means that (i) both $L$ and $R$ reverse order; and (ii) both $L R$ and $R L$ are closures, i.e.:

$$
\begin{aligned}
& \mathfrak{N} \subset L R \mathfrak{N} \quad \text { and } \quad L R L=L \\
& \mathfrak{N} \subset R L \mathfrak{N} \quad \text { and } \quad R L R=R .
\end{aligned}
$$

Let us denote by $\mathcal{F}^{L}$, resp. $\mathcal{F}^{R}$, the set of all $L R$-closed, resp. $R L$-closed, subspaces of $\mathfrak{A}$ :

$$
\begin{aligned}
& \mathcal{F}^{L}=\{\mathfrak{N} \subset \mathfrak{A}: \mathfrak{N}=L R \mathfrak{N}\}, \\
& \mathcal{F}^{R}=\{\mathfrak{N} \subset \mathfrak{A}: \mathfrak{N}=R L \mathfrak{N}\} .
\end{aligned}
$$

both ordered by inclusion. Then standard results from universal algebra yield the full multiplier structure of $\mathfrak{A}[4,6]$ :

Theorem 2.1 - Let $\mathfrak{A}$ be a partial *algebra and $\mathcal{F}^{L}$, resp. $\mathcal{F}^{R}$, the set of all LR-closed, resp. RL-closed, subspaces of $\mathfrak{A}$, both ordered by inclusion. Then 
(1) $\mathcal{F}^{L}$ is a complete lattice with lattice operations

$$
\mathfrak{M} \wedge \mathfrak{N}=\mathfrak{M} \cap \mathfrak{N}, \quad \mathfrak{M} \vee \mathfrak{N}=L R(\mathfrak{M}+\mathfrak{N})
$$

The largest element is $\mathfrak{A}$, the smallest $L \mathfrak{A}$.

(2) $\mathcal{F}^{R}$ is a complete lattice with lattice operations

$$
\mathfrak{M} \wedge \mathfrak{N}=\mathfrak{M} \cap \mathfrak{N}, \quad \mathfrak{M} \vee \mathfrak{N}=R L(\mathfrak{M}+\mathfrak{N})
$$

The largest element is $\mathfrak{A}$, the smallest $R \mathfrak{A}$.

(3) Both $L: \mathcal{F}^{R} \rightarrow \mathcal{F}^{L}$ and $R: \mathcal{F}^{L} \rightarrow \mathcal{F}^{R}$ are lattice anti-isomorphisms:

$$
L(\mathfrak{M} \wedge \mathfrak{N})=L \mathfrak{M} \vee L \mathfrak{N}, \text { etc. }
$$

(4) The involution $\mathfrak{N} \leftrightarrow \mathfrak{N}^{*}$ is a lattice isomorphism between $\mathcal{F}^{L}$ and $\mathcal{F}^{R}$.

In addition to the two lattices $\mathcal{F}^{L}$ and $\mathcal{F}^{R}$, it is useful to consider the subset $\mathcal{F}^{\Gamma} \subset \mathcal{F}^{L} \times \mathcal{F}^{R}$ consisting of matching pairs, that is:

$$
\mathcal{F}^{\Gamma}=\left\{(\mathfrak{N}, \mathfrak{M}) \in \mathcal{F}^{L} \times \mathcal{F}^{R}: \mathfrak{N}=L \mathfrak{M} \text { and } \mathfrak{M}=R \mathfrak{N}\right\}
$$

Indeed these pairs describe completely the partial multiplication of $\mathfrak{A}$, for we can write:

$$
(x, y) \in \Gamma \Longleftrightarrow \exists(\mathfrak{N}, \mathfrak{M}) \in \mathcal{F}^{\Gamma} \text { such that } x \in \mathfrak{N}, y \in \mathfrak{M} \text {. }
$$

\section{Topological partial *-algebras: Definition and first examples}

Let $\mathfrak{A}$ be a partial *-algebra with unit and assume it carries a locally convex, Hausdorff, topology $\tau$, which makes it into a locally convex topological vector space $\mathfrak{A}[\tau]$ (that is, the vector space operations are $\tau$-continuous). We denote by $\left\{p_{\alpha}\right\}$ a (directed) set of seminorms defining $\tau$.

As we saw in Section 2, the partial *-algebraic structure of $\mathfrak{A}$ is completely characterized by its spaces of left, resp. right, multipliers. Thus, quite naturally, we describe the topological structure of $\mathfrak{A}[\tau]$ by providing all spaces of multipliers with appropriate topologies.

We start with the following observation. Let $\mathfrak{M} \in \mathcal{F}^{R}$. To every $x \in L \mathfrak{M}$, one may associate a linear map $T_{x}^{L}$ from $\mathfrak{M}$ into $\mathfrak{A}$ :

$$
T_{x}^{L}(a)=x a, \quad a \in \mathfrak{M}, x \in L \mathfrak{M}
$$

This allows to define the topology $\rho_{\mathfrak{M}}$ on $\mathfrak{M}$ as the weakest locally convex topology on $\mathfrak{M}$ such that all maps $T_{x}^{L}, x \in L \mathfrak{M}$, are continuous from $\mathfrak{M}$ into $\mathfrak{A}[\tau]$. This is of course a 
projective topology. In the same way, the topology $\lambda_{\mathfrak{N}}$ on $\mathfrak{N} \in \mathcal{F}^{L}$ is the weakest locally convex topology on $\mathfrak{N}$ such that all maps $T_{y}^{R}: a \mapsto a y, y \in R \mathfrak{N}$, are continuous from $\mathfrak{N}$ into $\mathfrak{A}[\tau]$.

In terms of the seminorms $\left\{p_{\alpha}\right\}$ defining $\tau$, it is clear that the topology $\rho_{\mathfrak{M}}$ on $\mathfrak{M}$ may be defined by the seminorms

$$
p_{\alpha, \rho}^{x}(a)=p_{\alpha}(x a), x \in L \mathfrak{M}
$$

and the topology $\lambda_{\mathfrak{N}}$ on $\mathfrak{N}$ by the seminorms

$$
p_{\alpha, \lambda}^{y}(a)=p_{\alpha}(a y), y \in R \mathfrak{N} .
$$

It follows immediately from the definition that, whenever $\mathfrak{M}_{1}, \mathfrak{M}_{2} \in \mathcal{F}^{R}$ are such that $\mathfrak{M}_{1} \subset \mathfrak{M}_{2}$, then the topology $\rho_{\mathfrak{M}_{1}}$ is finer than the topology $\left(\rho_{\mathfrak{M}_{2}} \mid \mathfrak{M}_{1}\right)$ induced by $\mathfrak{M}_{2}$ on $\mathfrak{M}_{1}$. In other words, the embedding $\mathfrak{M}_{1} \rightarrow \mathfrak{M}_{2}$ is a continuous injection.

Take now $\mathfrak{A}$ itself. It carries three topologies, $\tau, \rho_{\mathfrak{A}}$ and $\lambda_{\mathfrak{A}}$. How do they compare? The topology $\rho_{\mathfrak{A}}$ makes all maps

$$
T_{x}^{L}: a \mapsto x a, \quad a \in \mathfrak{A}, x \in L \mathfrak{A}
$$

continuous. This is true in particular for $T_{e}^{L}$, where $e$ is the unit, which means precisely that the embedding $\mathfrak{A}\left[\rho_{\mathfrak{A}}\right] \rightarrow \mathfrak{A}[\tau]$ is continuous. The same applies of course to $\mathfrak{A}\left[\lambda_{\mathfrak{A}}\right] \rightarrow$ $\mathfrak{A}[\tau]$. In other words, both $\rho_{\mathfrak{A}}$ and $\lambda_{\mathfrak{A}}$ are finer than $\tau$.

As a consequence, since $\tau$ was assumed to be Hausdorff, all topologies $\rho_{\mathfrak{M}}, \mathfrak{M} \in \mathcal{F}^{R}$ and $\lambda_{\mathfrak{N}}, \mathfrak{N} \in \mathcal{F}^{L}$, are Hausdorff.

Now, for reasons of coherence, it would be preferable that all three topologies on $\mathfrak{A}$, $\tau, \rho_{\mathfrak{A}}$ and $\lambda_{\mathfrak{A}}$ be equivalent. Here is a handy criterion.

Lemma 3.1 - Let $\mathfrak{A}[\tau]$ be a partial *-algebra with locally convex topology $\tau$. Then:

(1) The projective topology $\rho_{\mathfrak{A}}$ on $\mathfrak{A}$ is equivalent to $\tau$ iff, for each $x \in L \mathfrak{A}$, the map $T_{x}^{L}: a \mapsto x a$ is continuous from $\mathfrak{A}[\tau]$ into itself.

(2) The projective topology $\lambda_{\mathfrak{A}}$ on $\mathfrak{A}$ is equivalent to $\tau$ iff, for each $y \in R \mathfrak{A}$, the map $T_{y}^{R}: a \mapsto$ ay is continuous from $\mathfrak{A}[\tau]$ into itself.

Proof. - (1) We know that $\rho_{\mathfrak{A}}>\tau$. Since $\rho_{\mathfrak{A}}$ is by definition the weakest topology on $\mathfrak{A}$ that makes the map $T_{x}^{L}$ continuous, the statement follows.

(2) Same argument.

Assume now that the involution $x \mapsto x^{*}$ is continuous in $\mathfrak{A}[\tau]$. If $\mathfrak{M} \in \mathcal{F}^{R}$ and $a \in \mathfrak{M}$, then $a^{*} \in \mathfrak{M}^{*}$, by Theorem 2.1 (4). Then, for $x \in R \mathfrak{M}^{*}$ and every seminorm $p_{\alpha}$ of $\mathfrak{A}[\tau]$, there is a seminorm $p_{\beta}$ such that, for some positive constant $c$,

$$
p_{\alpha, \lambda}^{x}\left(a^{*}\right)=p_{\alpha}\left(a^{*} x\right)=p_{\alpha}\left(\left(x^{*} a\right)^{*}\right) \leqslant c p_{\beta}\left(x^{*} a\right)=c p_{\beta, \rho}^{x^{*}}(a) .
$$


Similarly, if $\mathfrak{M}=\mathfrak{M}^{*} \in \mathcal{F}^{L} \cap \mathcal{F}^{R}, a=a^{*} \in \mathfrak{M}$ and $x \in L \mathfrak{M}$, we get

$$
p_{\alpha, \rho}^{x}\left(a^{*}\right)=p_{\alpha}(x a)=p_{\alpha}\left(\left(a x^{*}\right)^{*}\right) \leqslant c p_{\beta}\left(a x^{*}\right)=c p_{\beta, \lambda}^{x^{*}}(a) .
$$

Thus we have proven

Lemma 3.2 - Let $\mathfrak{A}[\tau]$ be a partial *-algebra with locally convex topology $\tau$. Assume that the involution $x \mapsto x^{*}$ is $\tau$-continuous. Then:

(1) For every $\mathfrak{M} \in \mathcal{F}^{R}$, the involution is continuous from $\mathfrak{M}\left[\rho_{\mathfrak{M}}\right]$ into $\mathfrak{M}^{*}\left[\lambda_{\mathfrak{M}} *\right] \in \mathcal{F}^{L}$.

(2) Let $\mathfrak{M}=\mathfrak{M}^{*} \in \mathcal{F}^{L} \cap \mathcal{F}^{R}$. Then the topology $\rho_{\mathfrak{M}}$ is equivalent to $\lambda_{\mathfrak{M}} *=\lambda_{\mathfrak{M}}$ on self-adjoint elements of $\mathfrak{M}$.

According to our goal to make the algebraic and the topological structure coincide as much as possible, on a topological partial *-algebra, we will naturally require that all three topologies $\rho_{a}, \lambda_{a}$ and $\tau$ coincide and that the involution be continuous. Let us now look at multiplier spaces $\mathfrak{M} \in \mathcal{F}^{R}$. If $\mathfrak{M}_{1} \subset \mathfrak{M}_{2}$, we have seen that the embedding is continuous. In order to make the structure tighter, we should also require that $\mathfrak{M}_{1}$ be dense in $\mathfrak{M}_{2}\left[\rho_{\mathfrak{M}_{2}}\right]$. This is true in many examples, typically the function spaces of Section 4 (such a condition is of course reminiscent of PIP-spaces - which these function spaces actually are also). Of course it is enough to require that $R \mathfrak{A}$ be dense in each $\mathfrak{M}\left[\rho_{\mathfrak{M}}\right] \in \mathcal{F}^{R}$. Indeed, if $R \mathfrak{A} \subset \mathfrak{M}_{1} \subset \mathfrak{M}_{2}$, and $R \mathfrak{A}$ is dense in $\mathfrak{M}_{2}$ for $\tau_{\mathfrak{M}_{2}}$, so is a fortiori $\mathfrak{M}_{1}$. But this condition is still too strong (and hardly verifiable in practice, because $\mathcal{F}^{R}$ is too large). To go beyond, we introduce the notion of generating family, a notion equivalent to that of rich subset for a compatibility relation, as described in [2].

Definition $3.3-A$ subset $\mathcal{I}^{R}$ of $\mathcal{F}^{R}$ is called a generating family if

(i) $R \mathfrak{A} \in \mathcal{I}^{R}$ and $\mathfrak{A} \in \mathcal{I}^{R}$.

(ii) $x \in L(y)$ iff $\exists \mathfrak{M} \in \mathcal{I}^{R}$ s.t. $y \in \mathfrak{M}, x \in L \mathfrak{M}$.

A generating family for $\mathcal{F}^{L}$ or $\mathcal{F}^{\Gamma}$ is defined in a similar way.

Clearly, if $\mathcal{I}^{R}$ is a generating family for $\mathcal{F}^{R}, \mathcal{I}^{L}=L \mathcal{I}^{R}=\left\{L \mathfrak{M}: \mathfrak{M} \in \mathcal{I}^{R}\right\}$ is generating for $\mathcal{F}^{L}$ and $\mathcal{I}^{\Gamma}=\mathcal{I}^{L} \times \mathcal{I}^{R}$ is generating for $\mathcal{F}^{\Gamma}$.

The usefulness of this notion is twofold :

(i) if $\mathcal{I}^{R}$ is generating for $\mathcal{F}^{R}$, so is the sublattice $\mathcal{J}^{R}$ of $\mathcal{F}^{R}$ generated from $\mathcal{I}^{R}$ by finite lattice operations $\vee$ and $\wedge$.

(ii) if $\mathcal{I}^{R}$ is generating, the complete lattice generated by $\mathcal{I}^{R}$ is $\mathcal{F}^{R}$ itself.

We make immediate use of this last property for weakening the density condition. 
Lemma 3.4 - Let $\mathfrak{A}[\tau]$ be a partial *-algebra with topology $\tau$. Assume there exists a generating family $\mathcal{I}^{R}$ for $\mathcal{F}^{R}$ such that $R \mathfrak{A}$ is dense in $\mathfrak{M}\left[\rho_{\mathfrak{M}}\right]$ for every $\mathfrak{M} \in \mathcal{I}^{R}$. Then, for any pair $\mathfrak{M}_{1}, \mathfrak{M}_{2} \in \mathcal{F}^{R}$ such that $\mathfrak{M}_{1} \subset \mathfrak{M}_{2}, \mathfrak{M}_{1}$ is dense in $\mathfrak{M}_{2}\left[\rho_{\mathfrak{M}_{2}}\right]$.

Proof. - Let $\mathfrak{M} \in \mathcal{F}^{R}$. Since $\mathcal{F}^{R}$ is the lattice completion of $\mathcal{I}^{R}$, we may write

$$
\mathfrak{M}=\bigcap_{\alpha} \mathfrak{N}_{\alpha}, \mathfrak{N}_{\alpha} \in \mathcal{I}^{R}, \mathfrak{N}_{\alpha} \supset \mathfrak{M}
$$

By assumption, $R \mathfrak{A}$ is dense in every $\mathfrak{N}_{\alpha}\left[\rho_{\mathfrak{N}_{\alpha}}\right]$. Then it is also dense in their intersection, endowed with the projective topology, since the latter is the projective limit of a directed set of subspaces [33]. But this is precisely $\mathfrak{M}\left[\rho_{\mathfrak{M}}\right]$.

Let now $\mathfrak{M}_{1} \subset \mathfrak{M}_{2}$, both in $\mathcal{F}^{R}$. Since $R \mathfrak{A}$ is dense in $\mathfrak{M}_{2}\left[\rho_{\mathfrak{M}_{2}}\right]$, so is $\mathfrak{M}_{1}$.

Putting all these considerations together, we may now state our definition of topological partial *-algebra.

Definition 3.5 - Let $\mathfrak{A}[\tau]$ be a partial *-algebra, which is a TVS for the locally convex topology $\tau$. Then $\mathfrak{A}[\tau]$ is called a topological partial *-algebra if the following two conditions are satisfied:

(i) the involution $a \mapsto a^{*}$ is $\tau$-continuous;

(ii) the maps $a \mapsto x a$ and $a \mapsto$ ay are $\tau$-continuous for all $x \in L \mathfrak{A}$ and $y \in R \mathfrak{A}$.

The topological partial *-algebra $\mathfrak{A}[\tau]$ is said to be tight, if, in addition,

(iii) there is a generating family $\mathcal{J}^{R}$ for $\mathcal{F}^{R}$ such that $R \mathfrak{A}$ is dense in $\mathfrak{M}\left[\rho_{\mathfrak{M}}\right]$ for each $\mathfrak{M} \in \mathcal{J}^{R}$.

As we shall see in the following sections, these conditions will be satisfied in most examples we consider. But before that, it is worth considering again the density condition (iii). According to Lemma 3.4, its effect is to ensure that all the embeddings $\mathfrak{M}_{1} \subset \mathfrak{M}_{2}\left(\mathfrak{M}_{1}, \mathfrak{M}_{2} \subset \mathcal{F}^{R}\right)$ have dense range. An equivalent statement would be that the dual of $\mathfrak{M}_{2}\left[\rho_{\mathfrak{M}_{2}}\right]$ be a subspace of the dual of $\mathfrak{M}_{1}\left[\rho_{\mathfrak{M}_{1}}\right]$. Thus we characterize these dual spaces.

Lemma 3.6 - Let $\mathfrak{M} \in \mathcal{F}^{R}$, with its projective topology $\rho_{\mathfrak{M}}$. Then a linear functional $F$ on $\mathfrak{M}$ is $\rho_{\mathfrak{M}}$-continuous if and only if it may be represented as

$$
F(x)=\sum_{i=1}^{n} G_{i}\left(a_{i} x\right),
$$

where each $G_{i}$ is a $\tau$-continuous functional on $\mathfrak{A}$ and $a_{i} \in L \mathfrak{M}, i=1 \ldots n$. 
Proof. - If $G$ is $\tau$-continuous and $a \in L \mathfrak{M}$, we get

$$
|G(a x)| \leqslant p(a x)
$$

where $p$ is a continuous seminorm on $\mathfrak{A}[\tau]$. It is clear that $p^{a}(x) \equiv p(a x)$ is a continuous seminorm on $\mathfrak{M}\left[\rho_{\mathfrak{M}}\right]$. Therefore, $F(x)=\sum_{i=1}^{n} G_{i}\left(a_{i} x\right), x \in \mathfrak{M}$, is $\rho_{\mathfrak{M}}$-continuous for $G_{i}$ and $a_{i}$ satisfying the assumptions.

Conversely, let $F$ be $\rho_{\mathfrak{M}}$-continuous on $\mathfrak{M}$. Then there exist seminorms $p_{\alpha_{1}}, \ldots, p_{\alpha_{n}}$, $a_{1}, \ldots, a_{n} \in L \mathfrak{M}$ and $c>0$ such that

$$
|F(x)| \leqslant c \sum_{i=1}^{n} p_{\alpha_{i}}\left(a_{i} x\right), x \in \mathfrak{M} .
$$

Let us consider the following subspace $\mathfrak{K}$ of $\mathfrak{A} \oplus \mathfrak{A} \ldots \oplus \mathfrak{A}(n$ terms):

$$
\mathfrak{K}=\left\{\left(a_{1} x, a_{2} x, \ldots, a_{n} x\right) \mid x \in \mathfrak{M}\right\} .
$$

Then the functional $G\left(\left(a_{1} x, a_{2} x, \ldots, a_{n} x\right)\right)=F(x)$ is linear and continuous on $\mathfrak{K}$ with respect to the product topology defined by $\tau$. By the Hahn-Banach theorem, $G$ can be extended to a continuous linear functional on $\mathfrak{A} \oplus \mathfrak{A} \ldots \oplus \mathfrak{A}$ ( $n$ terms). This implies that there exist linear functionals $G_{i}$ on $\mathfrak{A}$ such that $G\left(\left(Y_{1}, \ldots, Y_{n}\right)\right)=\sum_{i=1}^{n} G_{i}\left(Y_{i}\right)$. Therefore we conclude that $F(x)=\sum_{i=1}^{n} G_{i}\left(a_{i} x\right)$.

It is instructive to rewrite the form (3.1) in terms of tensor products :

$$
F=\sum_{i=1}^{n} G_{i} \otimes a_{i}, G_{i} \in \mathfrak{A}^{\prime}, a_{i} \in L \mathfrak{M}
$$

Then the statement of Lemma 3.6 may be reformulated as:

$$
\mathfrak{M}^{\prime}=\mathfrak{A}^{\prime} \otimes L \mathfrak{M} / \mathcal{K}\left[\mathfrak{M}^{\prime}\right]
$$

where the kernel $\mathcal{K}\left[\mathfrak{M}^{\prime}\right]$ consists of the forms in $\mathfrak{A}^{\prime} \otimes L \mathfrak{M}$ that vanish on $\mathfrak{M}$ :

$$
\mathcal{K}\left[\mathfrak{M}^{\prime}\right]=\left\{\sum_{1}^{n} G_{i} \otimes a_{i} \in \mathfrak{A}^{\prime} \otimes L \mathfrak{M}:\left(G_{i} \otimes a_{i}\right)(x)=0, \forall x \in \mathfrak{M}\right\}
$$

In this language, condition (iii) in Definition 3.5 says a sufficient condition for the embedding $\mathfrak{M}_{1} \subset \mathfrak{M}_{2}$ to have dense range is that

$$
\mathcal{K}\left[\mathfrak{M}^{\prime}\right]=\mathcal{K}\left[(R \mathfrak{A})^{\prime}\right] \cap\left(\mathfrak{A}^{\prime} \otimes L \mathfrak{M}\right), \forall \mathfrak{M} \in \mathcal{J}^{R}
$$

In other words, an element of $\mathfrak{A}^{\prime} \otimes L \mathfrak{M}$ vanishes on $\mathfrak{M}$ iff it vanishes on $R \mathfrak{A}$, which of course amounts to say that $\mathfrak{M}^{\prime}$ is a subspace of $(R \mathfrak{A})^{\prime}$. To see what can happen, it is 
amusing to consider the extreme case where $R \mathfrak{A}$ is one-dimensional, ie. $R \mathfrak{A}=\mathbb{C} e$. Then indeed one sees easily that $\mathcal{K}\left[\mathfrak{A}^{\prime}\right]=\{0\}$, whereas $\mathcal{K}\left[(R \mathfrak{A})^{\prime}\right]$ is of codimension 1 , and thus making $\mathfrak{M} \equiv \mathfrak{A}$ in $(\underline{3.2}), \mathcal{K}\left[\mathfrak{A}^{\prime}\right] \varsubsetneqq \mathcal{K}\left[(R \mathfrak{A})^{\prime}\right] \cap \mathfrak{A}^{\prime}$.

The previous discussion is summarized by the following

Proposition 3.7 - Let $\mathfrak{A}[\tau]$ be a topological partial ${ }^{*}$-algebra and $\mathcal{J}^{R}$ a generating family for $\mathcal{F}^{R}$. If the dual of each $\mathfrak{M}\left[\rho_{\mathfrak{M}}\right]$ can be identified with a subspace of $\left(R \mathfrak{A}\left[\rho_{R \mathfrak{A}}\right]\right)^{\prime}$, then $\mathfrak{A}[\tau]$ is a tight topological partial *-algebra.

Actually, the tightness condition, despite its appearance, is familiar in functional analysis. As we shall see in Section 4, many families of function spaces (such as $L^{p}$ spaces, Sobolev spaces, etc.) can be recast into topological partial *-algebras. Tightness, in these examples, simply expresses the existence of a space of universal multipliers which is dense in each one of the spaces of the family. This is often realized by suitable classes of $C^{\infty}$ functions.

As discussed in the Introduction, we feel that Definition 3.5 is natural, in the sense that it forces the topological structure determined by $\tau$ to be consistent with the multiplier structure of $\mathfrak{A}$.

As an illustration of the developed ideas, we consider two abstract examples.

\section{$3.1 \quad$ Topological quasi *-algebras}

Let $\left(\mathfrak{A}, \mathfrak{A}_{o}\right)$ be a topological quasi-algebra, that is, $\mathfrak{A}_{o}$ is a topological ${ }_{\text {-algebra such that }}$ the multiplication is separately, but not jointly, continuous for the topology of $\mathfrak{A}_{o}$ and the latter is not complete, and $\mathfrak{A}$ is the completion of $\mathfrak{A}_{o}$. Thus $\mathfrak{A}$ is only a partial *-algebra: the product $x y$ is defined only if either $x$ or $y$ belongs to $\mathfrak{A}_{o}$. Clearly, $\left(\mathfrak{A}, \mathfrak{A}_{o}\right)$ is a (trivial) partial ${ }^{*}$-algebra with $L \mathfrak{M}=R \mathfrak{M}=\mathfrak{A}_{o}$ and $\mathfrak{A}_{o}$ is dense in $\mathfrak{A}$. Every topological

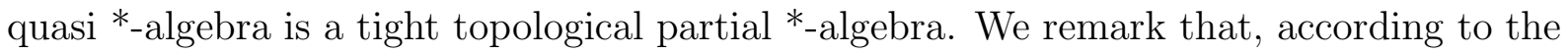
previous discussion, $\mathfrak{A}_{o}$ becomes in natural way a topological *-algebra with respect to the topology defined by the seminorms:

$$
p_{\alpha}^{x}(a)=\max \left\{p_{\alpha}(x a), p_{\alpha}(a x)\right\}, x \in \mathfrak{A},
$$

where the $p_{\alpha}$ 's are the seminorms defining the topology $\tau$ of $\mathfrak{A}$. This topology is finer than the initial topology of $A_{o}$.

\section{$3.2 C Q^{*}$-algebras}

This family of partial *-algebras appears, under several aspects, the natural extension of $\mathrm{C}^{*}$-algebras in the partial algebraic setting. The definition of $\mathrm{CQ}^{*}$-algebra that we will give here is different from the original one [15, 16], but fully equivalent. 
Definition 3.8 Let $\mathfrak{A}$ be a right Banach module over the $C^{*}$-algebra $\mathfrak{A}_{b}$, with isometric involution * and such that $\mathfrak{A}_{b} \subset \mathfrak{A}$. We say that $\left\{\mathfrak{A}, *, \mathfrak{A}_{b}, b\right\}$ is a $C Q^{*}$-algebra if

(i) $\mathfrak{A}_{b}$ is dense in $\mathfrak{A}$ with respect to its norm \|\|

(ii) $\mathfrak{A}_{o}:=\mathfrak{A}_{b} \cap \mathfrak{A}_{\sharp}$ is dense in $\mathfrak{A}_{b}$ with respect to its norm \|\|$_{b}$

(iii) $\|B\|_{b}=\sup _{A \in \mathfrak{A}}\|A B\|, \quad B \in \mathfrak{A}_{b}$

The following picture can be of some help in understanding the situation:

$\mathfrak{A}$

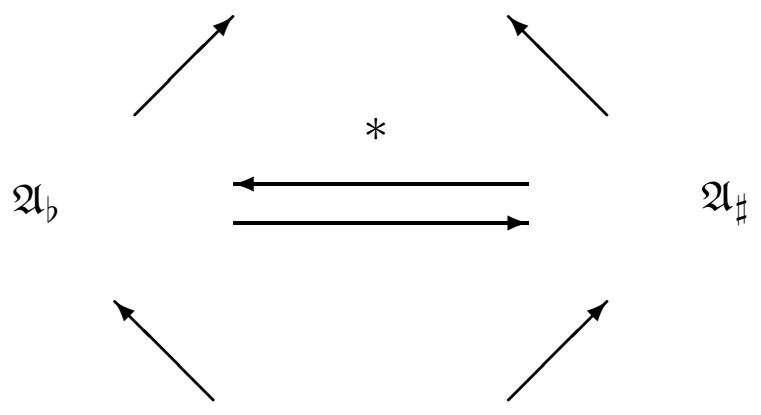

$\mathfrak{A}_{O}$

It is clear from the above definition that a $\mathrm{CQ}^{*}$-algebra is automatically a tight topological partial *-algebra.

To give the flavor of this construction, consider the following simple example [20]. Take a (Gel'fand) triplet of Hilbert spaces

$$
\mathcal{H}_{\lambda} \subset \mathcal{H} \subset \mathcal{H}_{\bar{\lambda}}
$$

where $\mathcal{H}_{\lambda}$ is, for instance, the domain of some self-adjoint operator $H>0$ (such that $(1+H)^{-1 / 2}$ is Hilbert-Schmidt) with the graph norm $\left\|(1+H)^{1 / 2} f\right\|$, and $\mathcal{H}_{\bar{\lambda}}$ is the antidual of $\mathcal{H}_{\lambda}$ with respect to the inner product of $\mathcal{H}$ (i.e. the norm on $\mathcal{H}_{\bar{\lambda}}$ is $\left\|(1+H)^{-1 / 2} f\right\|$ ). Then, if one makes the following identifications:

. $\mathfrak{A}=\mathcal{B}\left(\mathcal{H}_{\lambda}, \mathcal{H}_{\bar{\lambda}}\right)$, a Banach space;

. $R \mathfrak{A}=\mathcal{B}\left(\mathcal{H}_{\lambda}\right)$, a $\mathrm{C}^{*}$-algebra;

. $L \mathfrak{A}=\mathcal{B}\left(\mathcal{H}_{\bar{\lambda}}\right)$, also a $\mathrm{C}^{*}$-algebra; 


$$
\text { . } \mathfrak{A}_{o}=\mathcal{B}\left(\mathcal{H}_{\lambda}\right) \cap \mathcal{B}\left(\mathcal{H}_{\bar{\lambda}}\right)=\left\{A \in \mathcal{B}\left(\mathcal{H}_{\lambda}, \mathcal{H}_{\bar{\lambda}}\right): A \text { and } A^{*} \in \mathcal{B}\left(\mathcal{H}_{\lambda}\right)\right\}
$$

one can show that $\mathcal{B}\left(\mathcal{H}_{\lambda}, \mathcal{H}_{\bar{\lambda}}\right)$ is a $\mathrm{CQ}^{*}$-algebra and a tight topological partial *-algebra.

Due to its definition, a $\mathrm{CQ}^{*}$-algebra turns out to be useful in the description of certain quantum models in many cases where, for some physical reason, $R \mathfrak{A}$ is not large enough to include all the relevant observables of the given physical model, with their time-evolutes [17].

As for the structure, a $\mathrm{CQ}^{*}$-algebra can be viewed as the completion of a $\mathrm{C}^{*}$-algebra with respect to a weaker norm: this is exactly the case of proper $\mathrm{CQ}^{*}$-algebras $(R \mathfrak{A}=L \mathfrak{A} ; \sharp=$ b) [15] or even, under stronger assumptions, in the non-proper case [18]

Of particular interest is the case of a *-semisimple $\mathrm{CQ}^{*}$-algebra (i.e., with trivilal *_ radical). In this case, the analogy with $\mathrm{C}^{*}$-algebras becomes closer and closer.

First, for *-semisimple $\mathrm{CQ}^{*}$-algebras, it is possible to define a refinement of its partial multiplication: in this way, its lattices of multipliers become absolutely non-trivial. This allows an extension of certain facts of the familiar functional calculus for $\mathrm{C}^{*}$-algebras.

Second, the abelian case is completely understood: an abelian *-semisimple CQ*-algebra can be realized as a $\mathrm{CQ}^{*}$-algebra of functions by means of a generalized Gel'fand transform.

All these facts are discussed in [16, 19].

For all these reasons we consider $\mathrm{CQ}^{*}$-algebras, as a first step toward a more general study of partial $C^{*}$-algebras which is still to be carried out.

In the following two sections, we shall discuss in detail more sophisticated examples, namely functions spaces that will yield abelian topological partial *-algebra (Section 4) and partial *-algebras of operators (Section 5).

\section{Examples I : Topological partial *-algebras of functions}

\section{1. $L^{p}$ spaces on a finite interval}

A standard example of an abelian partial *-algebra [9] is the space $L^{1}([0,1], d x)$, equipped with the partial multiplication:

$$
f \in M(g) \Leftrightarrow \exists q \in[1, \infty] \text { such that } f \in L^{q}, g \in L^{\bar{q}}, 1 / q+1 / \bar{q}=1 \text {. }
$$

A similar structure may be given for every $L^{p}$. In fact one can show [19] that every space $L^{p}(X, d \mu)$, with $X$ a compact space and $\mu$ a Borel measure on $X$, is an abelian $\mathrm{CQ}^{*}$-algebra, with $\mathfrak{A}_{o}=\mathcal{C}(X)$, the space of continuous functions.

What we envisage here is the chain of all spaces $L^{p}$ at once, and for simplicity we take for $(X, \mu)$ the interval $[0,1]$ with Lebesgue measure. Thus we consider the chain 
$\mathcal{I}=\left\{L^{p}([0,1], d x), 1 \leqslant p \leqslant \infty\right\}$, with $L^{p} \subset L^{q}, p>q$. For $1<p<\infty$, every space $L^{p}$ is a reflexive Banach space with dual $L^{\bar{p}}(1 / p+1 / \bar{p}=1)$. Notice that duality in the sense of Banach spaces coincides with duality for the inner product of $L^{2}$ thanks to Hölder's inequality.

Now, being a chain, $\mathcal{I}$ is of course a lattice, albeit not a complete one. The lattice completion of $\mathcal{I}$, denoted $\mathcal{F}$, may be characterized explicity from the work of Davis et al. [24] (see also [2, 5]). Define the two spaces :

$$
L^{p-}=\bigcap_{1 \leqslant q<p} L^{q}, \quad L^{p+}=\bigcup_{p<q \leqslant \infty} L^{q}
$$

Then for $1<p \leqslant \infty, L^{p-}$, with the projective topology, is a non-normable reflexive Fréchet space, with dual $L^{\bar{p}+}$. And for $1 \leqslant p<\infty, L^{p+}$, with the inductive topology, is a nonmetrizable complete DF-space, with dual $L^{\bar{p}-}$ (a DF-space is the dual of a Fréchet space, necessarily non metrizable, unless the space and its dual are both Banach spaces [33]). Finally the following inclusions are strict:

$$
L^{p+} \subset L^{p} \subset L^{p-} \subset L^{q+} \quad(1<q<p<\infty)
$$

all embeddings in (4.2) are continuous and have dense range. Then the complete lattice $\mathcal{F}$ generated by $\mathcal{I}$ is also a chain, obtained by replacing each $L^{p}(1<p<\infty)$ by the corresponding triplet as in (4.2) and adding the two spaces $L^{\omega} \equiv L^{\infty-}$ (the so-called Arens space) and $L^{1+}$ :

$$
L^{\infty} \subset L^{\omega} \subset \ldots \subset L^{p+} \subset L^{p} \subset L^{p-} \subset \ldots \subset L^{1+} \subset L^{1} .
$$

Of course it would be more natural to index the spaces by $1 / p$, but traditions are respectable! Thus we take systematically our chains of spaces as increasing to the right, with decreasing $p$.

Now we turn to the partial *-algebra structure. The partial multiplication on the space $L^{1}([0,1], d x)$ is defined as in (4.1), i.e. $\mathcal{I}$ is a generating family. For computing multiplier spaces, define the following set, which characterizes the behavior of an individual vector $f \in L^{1}:$

$$
J(f)=\left\{q \geqslant 1: f \in L^{q}\right\}
$$

and let $p=\sup J(f)$, with $1 \leqslant p \leqslant \infty$.

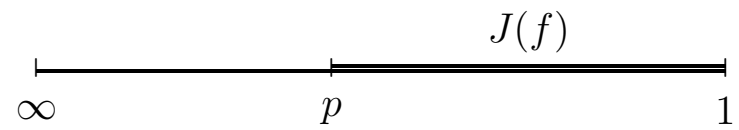

Figure 1 : The set $J(f)$. 
We distinguish two cases:

(i) $J(f)=[1, p]$, a closed interval, i.e. $f \in L^{p}$, but $f \notin L^{s}, \forall s>p$. Then it is easily seen that $M(f)=L^{\bar{p}}[19]$.

(ii) $J(f)=[1, p)$, a semi-open interval, i.e. $f \in L^{q}, \forall q<p$, hence $f \in L^{p-}=\bigcap_{q<p} L^{q}$, but $f \notin L^{p}$. Then $M(f)=\bigcup_{\bar{q}>\bar{p}} L^{\bar{p}}=L^{\bar{p}+}$.

From these results, it follows immediately that

$$
M L^{p}=L^{\bar{p}}, \quad M L^{p-}=L^{\bar{p}+}, \quad M L^{p+}=L^{\bar{p}-} .
$$

Notice that, if we define $f, g$ to be multiplicable whenever $f g \in L^{1}$, then the space of multipliers $M(f)$ of a given element $f$ is more complicated, but we still have $M L^{p}=L^{\bar{p}}$, etc, as follows from [19].

As for the multiplier topologies, we also have that

- $\rho_{L^{p}}$ is the $L^{p}$ norm topology

- $\rho_{L^{p-}}$ is the Fréchet projective topology on $L^{p-}$

- $\rho_{L^{p+}}$ is the DF topology on $L^{p+}$.

For both $\mathcal{I}$ and $\mathcal{F}$, the smallest space is $L^{\infty}=M L^{1}$, and it is dense in all the other ones. The involution $f \mapsto \bar{f}$ is of course $L^{1}$-continuous. The multiplication is continuous from $L^{\infty} \times L^{1}$ into $L^{1}$. In fact it is not only separately, but even jointly continuous and similarly from $L^{p} \times L^{\bar{p}}$ and from $L^{p-} \times L^{\bar{p}+}$ into $L^{1}$, thanks to Hölder's inequality and the fact that all topologies are either Fréchet or DF [33]. Since this result is general, we state it as a proposition.

Proposition 4.1 - Let $\mathfrak{A}[\tau]$ be a partial ${ }^{*}$-algebra with locally convex topology $\tau$, and $\mathcal{I}^{R}$ a generating family. Assume that:

(1) $\tau$ is a norm topology and $\mathfrak{A}[\tau]$ is a Banach space.

(2) Each space $\mathfrak{N} \in \mathcal{I}^{L}$, with the topology $\lambda_{\mathfrak{N}}$, and each space $\mathfrak{M} \in \mathcal{I}^{R}$, with the topology $\rho_{\mathfrak{M}}$, is a Banach space.

Then the multiplication is jointly continuous from $L \mathfrak{M} \times \mathfrak{M}$ into $\mathfrak{A}$ for every $\mathfrak{M} \in \mathcal{I}^{R}$, and one has

$$
\|a b\| \leqslant\|a\|_{L \mathfrak{M}}\|b\|_{\mathfrak{M}}, \forall a \in L \mathfrak{M}, b \in \mathfrak{M}
$$

The proof of (4.3) essentially reduces to the principle of uniform boundedness. Indeed, for fixed $a \in L \mathfrak{M}$, the map $a \mapsto T_{a}^{L}$ is continuous from $L \mathfrak{M}$ into the space of bounded operators on $\mathfrak{M}$, which gives $\|a b\| \leqslant c\|a\|_{L \mathfrak{M}}\|b\|_{\mathfrak{M}}$ for some constant $c>0$. The latter may then be eliminated by renormalizing all norms by a factor $c$. Notice that (4.3) is 
strongly reminiscent of a Hölder condition. In fact it reduces to the latter in the case of $L^{p}$ considered as a topological partial *-algebra, as discussed in Section 4 below.

A partial *-algebra that satisfies the conditions of Proposition 4.1 may be called a Banach partial *algebra, since the relation (4.3) is the analogue of the characteristic property of Banach algebras A similar result holds if one of the spaces $\mathfrak{M}, L \mathfrak{M}$ is a Fréchet space and the other a DF-space, with $\mathfrak{A}[\tau]$ itself a Fréchet space.

In conclusion, the topological structure, the PIP-space structure and the multiplier structure of $\mathcal{I}$ all coincide, and we have a tight topological partial *-algebra.

By the same token, we can consider every space $L^{p}$, as a topological partial *-algebra, simply by replacing the partial multiplication (4.1) by the following one:

$$
f \in M(g) \Leftrightarrow \exists r, s \in[p, \infty], 1 / r+1 / s=1 / p, \text { such that } f \in L^{r}, g \in L^{s}
$$

This amounts exactly to replace $\mathcal{I}$ or $\mathcal{F}$ by the (complete) sublattice indexed by $[p, \infty]$. The rest is identical.

\subsection{The spaces $L^{p}(\mathbb{R}, d x)$}

We turn now to the $L^{p}$ spaces on $\mathbb{R}$. If we consider the family $\left\{L^{p}(\mathbb{R}) \cap L^{1}(\mathbb{R}), 1 \leqslant p \leqslant \infty\right\}$, we obtain a scale similar to the previous one (except that the individual spaces are not complete), which may be used to endow $L^{1}(\mathbb{R})$ with the structure of a tight topological partial *-algebra.

However, the spaces $L^{p}(\mathbb{R})$ themselves no longer form a chain, no two of them being comparable. We have only

$$
L^{p} \cap L^{q} \subset L^{s}, \forall s \text { such that } p<s<q
$$

Hence we have to take the lattice generated by $\mathcal{I}=\left\{L^{p}(\mathbb{R}, d x), 1 \leqslant p \leqslant \infty\right\}$, that we call $\mathcal{J}$. The extreme spaces of the lattice are, respectively:

$$
V_{J}^{\#}=\bigcap_{1 \leqslant q \leqslant \infty} L^{q}, \quad \text { and } \quad V_{J}=\bigcup_{1 \leqslant q \leqslant \infty} L^{q}=\sum_{1 \leqslant q \leqslant \infty} L^{q} .
$$

Here too, the lattice structure allows to give to $V_{J}$ a structure of topological partial *-algebra, as we shall see now.

The lattice operations on $\mathcal{J}$ are easily described [2, 5, 22, 24]:

- $L^{p} \wedge L^{q}=L^{p} \cap L^{q}$ is a Banach space, with the projective (topology corresponding to the) norm

$$
\|f\|_{p \wedge q}=\|f\|_{p}+\|f\|_{q}
$$


- $L^{p} \vee L^{q}=L^{p}+L^{q}$ is a Banach space, with the inductive (topology corresponding to the) norm

$$
\|f\|_{p \vee q}=\inf _{f=g+h}\left(\|g\|_{p}+\|h\|_{q}\right), g \in L^{p}, h \in L^{q} .
$$

- For $1<p, q<\infty$, both spaces $L^{p} \wedge L^{q}$ and $L^{p} \vee L^{q}$ are reflexive and $\left(L^{p} \wedge L^{q}\right)^{\prime}=$ $L^{\bar{p}} \vee L^{\bar{q}}$.

At this stage, it is convenient to introduce a unified notation:

$$
L^{(p, q)}= \begin{cases}L^{p} \wedge L^{q}, & \text { if } p \geqslant q, \\ L^{p} \vee L^{q}, & \text { if } p \leqslant q .\end{cases}
$$

Thus, for $1<p, q<\infty$, each space $L^{(p, q)}$ is a reflexive Banach space, with dual $L^{(\bar{p}, \bar{q})}$. The modifications when $p, q$ equal 1 or $\infty$ are obvious.

Next, if we represent $(p, q)$ by the point of coordinates $(1 / p, 1 / q)$, we may associate all the spaces $L^{(p, q)}(1 \leqslant p, q \leqslant \infty)$ in a one-to-one fashion with the points of a unit square $J=[0,1] \times[0,1]$ (see Figure 2). Thus, in this picture, the spaces $L^{p}$ are on the main diagonal, intersections $L^{p} \cap L^{q}$ above it and sums $L^{p}+L^{q}$ below. The space $L^{(p, q)}$ is contained in $L^{\left(p^{\prime}, q^{\prime}\right)}$ if $(p, q)$ is on the left and/or above $\left(p^{\prime}, q^{\prime}\right)$. Thus the smallest space $L^{(\infty, 1)}=L^{\infty} \cap L^{1}$ corresponds to the upper left corner, the largest one, $L^{(1, \infty)}=L^{1}+L^{\infty}$, to the lower right corner. Inside the square, duality corresponds to symmetry with respect to the center $(1 / 2,1 / 2)$ of the square, which represents the space $L^{2}$. 


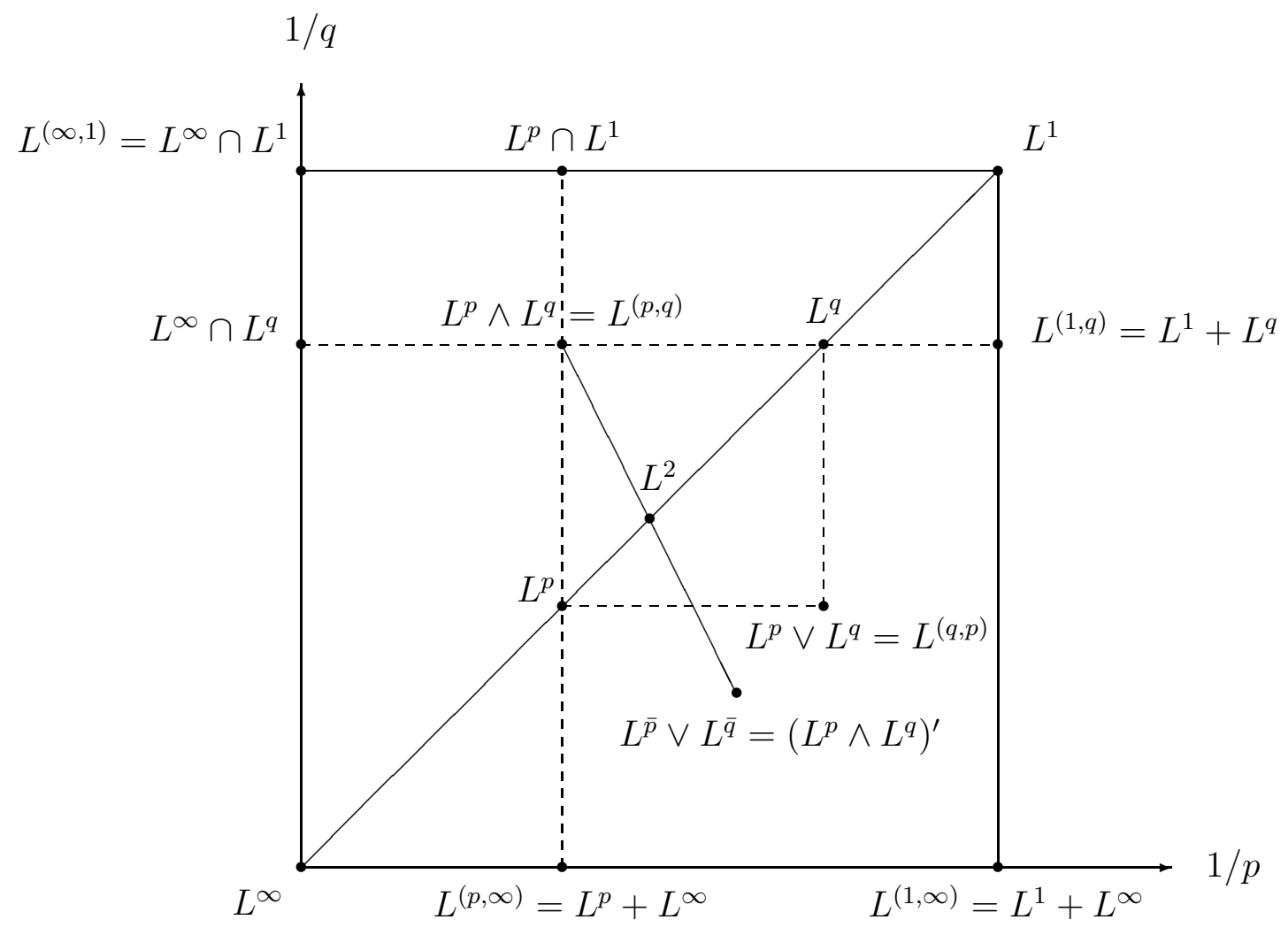

Figure 2: The unit square describing the lattice $J$

The ordering of the spaces corresponds to the following rule:

$$
L^{(p, q)} \subset L^{\left(p^{\prime}, q^{\prime}\right)} \quad \Longleftrightarrow \quad(p, q) \leqslant\left(p^{\prime}, q^{\prime}\right) \quad \Longleftrightarrow \quad p \geqslant p^{\prime} \text { and } q \leqslant q^{\prime} .
$$

For $\infty>q_{o}>1$, consider now the horizontal row $q=q_{o},\left\{L^{\left(p, q_{o}\right)}: \infty>p>1\right\}$. It corresponds to the chain:

$$
\begin{array}{r}
\ldots \subset L^{r} \cap L^{q_{o}} \subset \ldots \subset L^{q_{o}} \subset \ldots \subset L^{s}+L^{q_{o}} \subset \ldots \\
\left(\infty>r>q_{o}>s>1\right)
\end{array}
$$

sitting between the extreme elements $L^{\infty} \cap L^{q_{o}}$ on the left and $L^{1}+L^{q_{o}}$ on the right. The point is that all the embeddings in the chain (4.6) are continuous and have dense range.

The same holds true for a vertical row $p=p_{o},\left\{L^{\left(p_{o}, q\right)}: 1<q<\infty\right\}$ :

$$
\begin{array}{r}
\ldots \subset L^{p_{o}} \cap L^{s} \subset \ldots \subset L^{p_{o}} \subset \ldots \subset L^{p_{o}}+L^{r} \subset \ldots \\
\\
\left(1<s<p_{o}<r<\infty\right)
\end{array}
$$


Combining these two facts, we see that the partial order extends to the spaces $L^{(p, q)}(1<$ $p, q<\infty)$, inclusion meaning now continuous embedding with dense range.

Now the set of points contained in the square $J$ may be considered as an involutive lattice with respect to the partial order (4.5), with operations:

$$
\begin{aligned}
(p, q) \wedge\left(p^{\prime}, q^{\prime}\right) & =\left(p \vee p^{\prime}, q \wedge q^{\prime}\right) \\
(p, q) \vee\left(p^{\prime}, q^{\prime}\right) & =\left(p \wedge p^{\prime}, q \vee q^{\prime}\right) \\
\overline{(p, q)} & =(\bar{p}, \bar{q}),
\end{aligned}
$$

where, as usual, $p \wedge p^{\prime}=\min \left\{p, p^{\prime}\right\}, p \vee p^{\prime}=\max \left\{p, p^{\prime}\right\}$.

The considerations made above imply that the lattice $\mathcal{J}$ generated by $\mathcal{I}=\left\{L^{p}\right\}$ is already obtained at the first generation. For example, $L^{(r, s)} \wedge L^{(a, b)}=L^{(r \vee a, s \wedge b)}$ (see Figure 3 ), and the latter may be either above, on or below the diagonal, depending on the values of the indices. For instance, if $p<q<s$, then $L^{(p, q)} \wedge L^{(q, s)}=L^{q}$, both as sets and as topological vector spaces.

The conclusion is that, using this language, the only difference between the two cases $\left\{L^{p}([0,1])\right\}$ and $\left\{L^{p}(\mathbb{R})\right\}$ lies in the type of order obtained: a chain $I$ (total order) or a partially ordered lattice $J$. From this remark, the lattice completion of $\mathcal{J}$ can be obtained exactly as before, using the results of [24]. This introduces again Fréchet and DF-spaces, all reflexive if we start from $1<p<\infty$, and in natural duality as in the previous case. In particular, for the spaces of the first 'generation', it suffices to consider intervals $S \subset[1, \infty]$ and define the spaces

$$
L^{P}(S)=\bigcap_{q \in S} L^{q}, \quad L^{I}(S)=\bigcup_{q \in S} L^{q}
$$

Then:

- If $S$ is a closed interval $S=[p, q]$, with $p<q$, then $L^{P}(S)=L^{p} \wedge L^{q}=L^{(q, p)}$ and $L^{I}(S)=L^{p} \vee L^{q}=L^{(p, q)}$ are Banach spaces. If $S$ is a semi-open or open interval, $L^{P}(S)$ is a non-normable Fréchet space and $L^{I}(S)$ a DF-space.

- Let $S \subset(1, \infty)$ and define $\bar{S}=\{\bar{q}: q \in S\}$. Then $\left(L^{P}(S)\right)^{\prime}=L^{I}(\bar{S}), \quad\left(L^{I}(S)\right)^{\prime}=$ $L^{P}(\bar{S})$ 


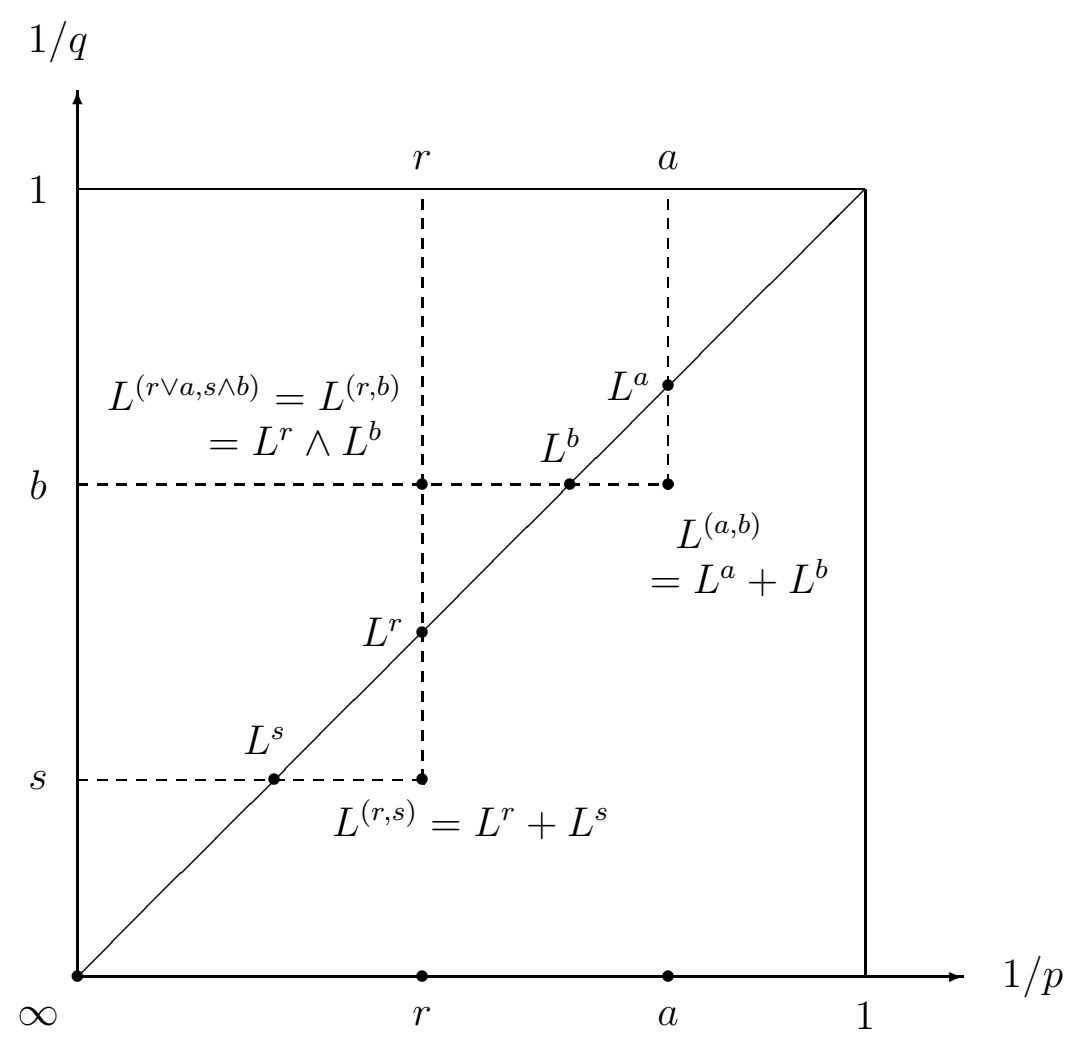

Figure 3 : The intersection of two spaces from $J$.

A special rôle will be played in the sequel by the spaces $L^{I}$ corresponding to semi-infinite intervals, namely:

$$
\begin{aligned}
& L^{(p, \infty)}=L^{I}([p, \infty])=\bigcup_{p \leqslant s \leqslant \infty} L^{s}=L^{p}+L^{\infty}, \text { which is a nonreflexive Banach space. } \\
& L^{(p, \omega)}=L^{I}([p, \infty))=\bigcup_{p \leqslant s<\infty} L^{s}, \text { which is a reflexive DF-space. }
\end{aligned}
$$

As for the lattice completion $\mathcal{F}$, one can essentially repeat the argument of [2, Example 3.B] and build an 'enriched' or 'nonstandard' square $F$, exactly as in the previous section. Take first $1<q<\infty$, that is, the interior $J_{o}$ of the square $J$. The extreme spaces of the corresponding complete lattice $\mathcal{F}_{o}$ are:

$$
V_{o}^{\#}=\bigcap_{1<q<\infty} L^{q}, \quad \text { and } \quad V_{o}=\bigcup_{1<q<\infty} L^{q}=\sum_{1<q<\infty} L^{q},
$$

with their projective and inductive topologies, respectively. All embeddings are continuous and have dense range. Thus the space $V_{o}$, together with either of the two lattices $\mathcal{J}_{o}=$ 
$\left\{V_{\alpha}, \alpha \in J_{o}\right\}$ or $\mathcal{F}_{o}=\left\{V_{\alpha}, \alpha \in F_{o}\right\}$, is a PIP-space, with the usual $L^{2}$ inner product and $\left(V_{\alpha}\right)^{\#}=\left(V_{\alpha}\right)^{\times}=\left(V_{\bar{\alpha}}\right)$.

Similar results are valid when one includes $L^{1}$ and $L^{\infty}$, except for the obvious modifications concerning duality. The extreme spaces of the full lattice $\mathcal{F}$ then are $V_{J}^{\#}=L_{\rho}^{\#}=$ $L^{1} \cap L^{\infty}$ and $V_{J}=L_{\rho}=L^{1}+L^{\infty}$, with their projective and inductive norms, which make them into nonreflexive Banach spaces (none of them is the dual of the other). Notice that the space $L_{\rho}$, originally introduced by Gould [39], contains strictly all the $L^{p}, 1 \leqslant p \leqslant \infty$.

We turn now to the partial *-algebra structure on $V_{J}$. Again we start from the lattice $\mathcal{J}$, which is generating for the PIP-space structure. The basic fact is Hölder's inequality, which says that pointwise multiplication is continuous from $L^{p} \times L^{q}$ into $L^{r}$, where $1 / p+$ $1 / q=1 / r$. From this we can compute the multipliers of all the elements of $\mathcal{J}$ in several steps (as usual we write $p \wedge q=\min \{p, q\}, p \vee q=\max \{p, q\}$ ):

- $L^{s} \subset M L^{p}$ iff $\bar{p} \leqslant s \leqslant \infty$. Thus

$$
M L^{p}=\bigcup_{s \geqslant \bar{p}} L^{s}=L^{(\bar{p}, \infty)} .
$$

- Let $p>q$, so that $L^{(p, q)}=L^{p} \wedge L^{q}$. Then

$$
M L^{(p, q)}=M L^{p} \vee M L^{q}=L^{(\bar{p}, \infty)} \vee L^{(\bar{q}, \infty)}=L^{(\bar{p}, \infty)}
$$

- Let $p<q$, so that $L^{(p, q)}=L^{p} \vee L^{q}$. Then

$$
M L^{(p, q)}=M L^{p} \wedge M L^{q}=L^{(\bar{p}, \infty)} \wedge L^{(\bar{q}, \infty)}=L^{(\bar{q}, \infty)} .
$$

- Thus in all cases

$$
M L^{(p, q)}=L^{(\bar{p} \wedge \bar{q}, \infty)}=L^{(\overline{p \vee q}, \infty)} .
$$

If one does not want to include $L^{\infty}$, one simply replaces (4.8) by

$$
M L^{(p, q)}=L^{(\bar{p} \wedge \bar{q}, \omega)}=L^{(\overline{p \vee q}, \omega)} .
$$

Applying the rule (4.8) or(4.9) twice, one gets immediately $M M L^{(p, q)}=L^{(p \vee q, \infty)}$, resp. $M M L^{(p, q)}=L^{(p \vee q, \omega)}$.

In conclusion, the generating family for multiplication is the set $\mathcal{J}^{M}=\left\{L^{(p, \infty)}, 1 \leqslant\right.$ $p \leqslant \infty$, corresponding to the bottom side of the square $J$ in Figure 2, and it is a chain of Banach spaces, exactly as in the case of the $L^{p}$ spaces over $[0,1]$. Thus we write the partial multiplication on $L^{1}+L^{\infty}$ as:

$$
f \in M(g) \Leftrightarrow \exists q \in[1, \infty] \text { such that } f \in L^{(q, \infty)}, g \in L^{(\bar{q}, \infty)}, 1 / q+1 / \bar{q}=1
$$


and that on $V$ as:

$$
f \in M(g) \Leftrightarrow \exists q \in(1, \infty) \text { such that } f \in L^{(q, \omega)}, g \in L^{(\bar{q}, \omega)}, 1 / q+1 / \bar{q}=1,
$$

Finally, we can immediately conclude that the complete lattice $\mathcal{F}^{M}$ is the 'enriched' chain $\mathcal{F}^{M}=\left\{L^{(p \epsilon, \infty)}, p \epsilon=p-, p\right.$ or $\left.p+, 1 \leqslant p \leqslant \infty\right\}$, and similarly with $\omega$ instead of $\infty$.

Exactly as in the case of a finite interval, we may restrict the generating spaces to $\left\{L^{s}, p \leqslant s \leqslant \infty\right)$, which amounts to take a subsquare of $J$. The rest is obvious.

Another interesting structure of partial *-algebra may be given to the spaces $L_{\rho}$ or $V$, simply replacing multiplication by convolution. According to Hausdorff-Young's inequality, convolution maps $L^{p} \times L^{q}$ continuously into $L^{r}$, where $1 / p+1 / q=1+1 / r$. From this we can compute the multipliers of all the elements of $\mathcal{J}$ as in the previous case (to avoid confusion, we use here the notation $M_{*}$ ):

- $L^{s} \subset M_{*} L^{p}$ iff $s \leqslant \bar{p}$. Thus

$$
M_{*} L^{p}=\bigcup_{s \leqslant \bar{p}} L^{s}=L^{(1, \bar{p})}=L^{1}+L^{\bar{p}}
$$

- For $p>q, M_{*} L^{(p, q)}=L^{(1, \bar{p})}$, and for $p<q, M_{*} L^{(p, q)}=L^{(1, \bar{q},)}$.

- Thus in all cases

$$
M_{*} L^{(p, q)}=L^{(1, \bar{p} \wedge \bar{q})}=L^{(1, \overline{p \vee q})} .
$$

Again these multiplier spaces constitute a chain, this one corresponding to the right-hand side of the square $J$.

\subsection{Amalgam spaces}

The lesson of the previous example is that an involutive lattice of (preferably reflexive) Banach spaces (that is, a PIP-space of type B or $\mathrm{H}$ [3]) turns quite naturally into a (tight) topological partial *-algebra if it possesses a partial multiplication that verify a (generalized) Hölder inequality. A whole class of examples is given by the so-called amalgam spaces first introduced by N. Wiener [38] and developed systematically by Holland [26]. The simplest ones are the spaces $\left(L^{p}, \ell^{q}\right)$ (sometimes denoted $W\left(L^{p}, \ell^{q}\right)$ ) consisting of functions on $\mathbb{R}$ which are locally in $L^{p}$ and have $\ell^{q}$ behavior at infinity, in the sense that the $L^{p}$ norms over the intervals $(n, n+1)$ form an $\ell^{q}$ sequence (see the review paper [25]). For $1 \leqslant p, q<\infty$, the norm

$$
\|f\|_{p, q}=\left\{\sum_{n=-\infty}^{\infty}\left[\int_{n}^{n+1}|f(x)|^{p} d x\right]^{q / p}\right\}^{1 / q}
$$


makes $\left(L^{p}, \ell^{q}\right)$ into a Banach space. The same is true for the obvious extensions to $p$ and/or $q$ equal to 1 or $\infty$. Notice that $\left(L^{p}, \ell^{p}\right)=L^{p}$. The spaces $\left(L^{p}, \ell^{q}\right)$ have many interesting applications, for instance in the context of various Tauberian theorems. New ones have been found recently in the theory of frames (nonorthogonal expansions) 21].

These spaces obey the following (immediate) inclusion relations, with all embeddings continuous:

- If $q_{1} \leqslant q_{2}$, then $\left(L^{p}, \ell^{q_{1}}\right) \subset\left(L^{p}, \ell^{q_{2}}\right)$.

- If $p_{1} \leqslant p_{2}$, then $\left(L^{p_{2}}, \ell^{q}\right) \subset\left(L^{p_{1}}, \ell^{q}\right)$.

From this it follows that the smallest space is $\left(L^{\infty}, \ell^{1}\right)$ and the largest one is $\left(L^{1}, \ell^{\infty}\right)$, and therefore

- If $p \geqslant q$, then $\left(L^{p}, \ell^{q}\right) \subset L^{p} \cap L^{q} \subset L^{s}, \forall q<s<p$.

- If $p \leqslant q$, then $\left(L^{p}, \ell^{q}\right) \supset L^{p} \cup L^{q}$.

Once again, Hölder's inequality is satisfied. Whenever $f \in\left(L^{p}, \ell^{q}\right)$ and $g \in\left(L^{\bar{p}}, \ell^{\bar{q}}\right)$, then $f g \in L^{1}$ and one has

$$
\|f g\|_{1} \leqslant\|f\|_{p, q}\|g\|_{\bar{p}, \bar{q}} .
$$

Therefore, one has the expected duality relation:

$$
\left(L^{p}, \ell^{q}\right)^{\prime}=\left(L^{\bar{p}}, \ell^{\bar{q}}\right), \text { for } 1 \leqslant q, p<\infty .
$$

The interesting fact is that, for $1 \leqslant p, q \leqslant \infty$, the set $\mathcal{J}$ of all amalgam spaces $\left\{\left(L^{p}, \ell^{q}\right)\right\}$ may be represented by the points $(p, q)$ of the same unit square $J$ as in the previous example, with the same order structure. In particular, $\mathcal{J}$ is a lattice with respect to the order (4.5):

$$
\begin{aligned}
& \left(L^{p}, \ell^{q}\right) \wedge\left(L^{p^{\prime}}, \ell^{q^{\prime}}\right)=\left(L^{p \vee p^{\prime}}, \ell^{q \wedge q^{\prime}}\right) \\
& \left(L^{p}, \ell^{q}\right) \vee\left(L^{p^{\prime}}, \ell^{q^{\prime}}\right)=\left(L^{p \wedge p^{\prime}}, \ell^{q \vee q^{\prime}}\right),
\end{aligned}
$$

where again $\wedge$ means intersection with projective norm and $\vee$ means vector sum with inductive norm.

We turn now to the partial *-algebra structure of $\mathcal{J}$. At first sight, the situation becomes different, because whereas $L^{1}$ is a partial *-algebra, $\ell^{\infty}$ is an algebra under componentwise multiplication, $\left(a_{n}\right) \cdot\left(b_{n}\right)=\left(a_{n} b_{n}\right)$. The $L^{p}$ component characterizes the local behavior. Hence,

$$
M\left(L^{p}, \ell^{q}\right) \supset\left(L^{\bar{p}}, \ell^{\infty}\right), \forall q,
$$


and since the latter are totally ordered, we obtain, exactly as in the cases of the $L^{p}$ spaces:

$$
M\left(L^{p}, \ell^{q}\right)=\left(L^{\bar{p}}, \ell^{\infty}\right)
$$

Thus the natural partial multiplication on $\mathcal{J}$ reads:

$$
f \in M(g) \Leftrightarrow \exists p \in[1, \infty] \text { such that } f \in\left(L^{p}, \ell^{\infty}\right) \text { and } g \in\left(L^{\bar{p}}, \ell^{\infty}\right) \text {. }
$$

The rest is as before, including the identification of the complete lattice $\mathcal{F}$ with the 'enriched' interval $[1, \infty]$.

Since the amalgam spaces $\left(L^{p}, \ell^{q}\right)$ obey the same Hausdorff-Young inequality as the $L^{p}$ spaces, we may obtain here too another structure of partial *-algebra with the convolution as partial multiplication. Let $f \in\left(L^{p}, \ell^{q}\right)$ and $g \in\left(L^{p^{\prime}}, \ell^{q^{\prime}}\right)$, with $1 / p+1 / p^{\prime} \geqslant 1,1 / q+$ $1 / q^{\prime} \geqslant 1$, that is, $p^{\prime} \leqslant \bar{p}, q^{\prime} \leqslant \bar{q}$. Then $f * g \in\left(L^{p^{\prime \prime}}, \ell^{q^{\prime \prime}}\right)$, with $1 / p^{\prime \prime}=1 / p+1 / p^{\prime}-1,1 / q^{\prime \prime}=$ $1 / q+1 / q^{\prime}-1$. By the same arguments as in the previous section, we obtain

$$
M_{*}\left(L^{p}, \ell^{q}\right)=\left(L^{1}, \ell^{\bar{p} \wedge \bar{q}}\right)=\left(L^{1}, \ell^{\overline{p \vee q}}\right)
$$

As before, these multiplier spaces constitute a chain, corresponding to the right-hand side of the square $J$.

\section{Examples II: Topological partial *-algebras of operators}

\subsection{Operators on a lattice of Hilbert spaces}

Our first example is the partial *-algebra of operators on a lattice of Hilbert spaces (LHS), also called indexed PIP-spaces of type $(\mathrm{H})[3]$. By this we mean a vector space $V$ together with a family of subspaces $V_{I}=\left\{\mathcal{H}_{r}, r \in I\right\}$, where

- $V=\sum_{r \in I} \mathcal{H}_{r}$

- the index set $I$ is an involutive lattice with order-reversing involution $r \leftrightarrow \bar{r}$ (that is, $p \leqslant q$ implies $\bar{q} \leqslant \bar{p}$ and $\overline{\bar{p}}=p$ ) and a unique element $o$ such that $\bar{o}=o$;

- each $\mathcal{H}_{r}$ is a Hilbert space with norm $\|\cdot\|_{r}$ and $\mathcal{H}_{\bar{r}}=\mathcal{H}_{r}^{\times}$, the anti-dual of $\mathcal{H}_{r}$; in particular, $\mathcal{H}_{\bar{o}}=\mathcal{H}_{o}^{\times}=\mathcal{H}_{o}$

- the family $V_{I}$ is an involutive lattice under set inclusion and lattice operations

. $\mathcal{H}_{p \wedge q}=\mathcal{H}_{p} \cap \mathcal{H}_{q}$, with the projective norm $\|f\|_{p \wedge q}^{2}=\|f\|_{p}^{2}+\|f\|_{q}^{2}$,

. $\mathcal{H}_{p \vee q}=\mathcal{H}_{p}+\mathcal{H}_{q}$, with the inductive norm $\|f\|_{p \vee q}^{2}=\inf _{f=g+h}\left(\|g\|_{p}^{2}+\|h\|_{q}^{2}\right)$, $\left(g \in \mathcal{H}_{p}, f \in \mathcal{H}_{q}\right)$ 
(we use squared norms in these definitions in order to get Hilbert norms for the projective and inductive ones).

- The inner product of $\mathcal{H}_{o}$ extends to a partial inner product $\langle\cdot \mid \cdot\rangle$, that is, a Hermitian sesquilinear form defined exactly on dual pairs $\mathcal{H}_{r}, \mathcal{H}_{\bar{r}}$.

It follows that $(V,\langle\cdot \mid \cdot\rangle)$ is a PIP-space, and $V^{\#}=\bigcap_{r \in I} \mathcal{H}_{r}$. We assume the partial inner product to be nondegenerate, that is, $\left(V^{\#}\right)^{\perp}=\{0\}$, which means that $\langle f \mid g\rangle=0, \forall f \in$ $V^{\#}$, implies $g=0$. This entails that $\left(V^{\#}, V\right)$, as well as every pair $\left(\mathcal{H}_{r}, \mathcal{H}_{\bar{r}}\right)$, is a dual pair in the sense of topological vector space theory [33. Note that the Mackey topology $\tau\left(\mathcal{H}_{r}, \mathcal{H}_{\bar{r}}\right)$ on $\mathcal{H}_{r}$ coincides with the original norm topology.

Once again the topological and lattice structures coincide: $q<p$ implies $\mathcal{H}_{q} \subset \mathcal{H}_{p}$ and the embedding is continuous with dense range. Similarly, $\mathcal{H}_{p \wedge q}$ and $\mathcal{H}_{\bar{p} \vee \bar{q}}$ are dual to each other. Moreover, $V^{\#}$ is dense in every $\mathcal{H}_{r}, r \in I$.

Typical examples of LHS are:

(i) Hilbert scales

Many examples of Hilbert scales, discrete or continuous, appear in applications. For instance:

. The scale built on the powers of a positive self-adjoint operator $H>1: \mathcal{H}_{n}=$ $D\left(H^{n}\right)$, with the graph norm $\|f\|_{n}=\left\|H^{n} f\right\|, n \in \mathbb{N}$, and $\mathcal{H}_{-n}=\mathcal{H}_{n}^{\times}$.

- The scale of Sobolev spaces $W_{s}^{2}(\mathbb{R}), s \in \mathbb{R}$, where $f \in W_{s}^{2}(\mathbb{R})$ if its Fourier transform $\widehat{f}$ satisfies the condition $\left(1+|.|^{2}\right)^{s / 2} \widehat{f} \in L^{2}(\mathbb{R})$. The norm is $\|f\|_{s}=$ $\left\|\left(1+|.|^{2}\right)^{s / 2} \widehat{f}\right\|, s \in \mathbb{R}$. Of course, similar considerations hold for the Banach scale $\left\{W_{s}^{p}(\mathbb{R}), s \in \mathbb{R}\right\}, 1<p<\infty$, but here we restrict ourselves to the Hilbert case $p=2$.

We will come back to these two examples at the end of this Section 5 .

(ii) Weighted $\ell^{2}$ sequence spaces

Given a sequence of positive numbers, $r=\left(r_{n}\right), r_{n}>0$, define $\ell^{2}(r)=\left\{x=\left(x_{n}\right)\right.$ : $\left.\sum_{n=1}^{\infty}\left|x_{n}\right|^{2} r_{n}^{-1}<\infty\right\}$. The lattice operations read:

. involution: $\ell^{2}(\bar{r})=\ell^{2}(r)^{\times}, \bar{r}_{n}=1 / r_{n}$.

. infimum: $\ell^{2}(p) \wedge \ell^{2}(q)=\ell^{2}(r), r_{n}=\min \left(p_{n}, q_{n}\right)$.

. supremum: $\ell^{2}(p) \vee \ell^{2}(q)=\ell^{2}(s), s_{n}=\max \left(p_{n}, q_{n}\right)$.

As for the extreme spaces, it is easy to see that the family $\left\{\ell^{2}(r)\right\}$ generates the space $\omega$ of all complex sequences, while the intersection is the space $\varphi$ of all finite sequences. 
(iii) Weighted $L^{2}$ function spaces

Instead of sequences, we consider locally integrable (i.e. integrable on bounded sets) functions $f \in L_{\mathrm{loc}}^{1}(\mathbb{R}, d x)$ and define again weighted spaces:

$$
\begin{aligned}
I & =\left\{r \in L_{\mathrm{loc}}^{1}(\mathbb{R}, d x): r(x)>0, \text { a.e. }\right\} \\
L^{2}(r) & =\left\{f \in L_{\mathrm{loc}}^{1}(\mathbb{R}, d x): \int|f(x)|^{2} r(x)^{-1} d x<\infty\right\}, r \in I .
\end{aligned}
$$

Then we get exactly the same structure as in (ii):

. involution: $L^{2}(r) \Leftrightarrow L^{2}(\bar{r}), \bar{r}=1 / r$.

. infimum: $L^{2}(p) \wedge L^{2}(q)=L^{2}(r), r(x)=\min (p(x), q(x))$.

. supremum: $L^{2}(p) \vee L^{2}(q)=L^{2}(s), s(x)=\max (p(x), q(x))$.

. extreme spaces:

$$
\bigcup_{r \in I} L^{2}(r)=L_{\mathrm{loc}}^{1}, \quad \bigcap_{r \in I} L^{2}(r)=L_{\mathrm{c}}^{\infty}
$$

where $L_{\mathrm{c}}^{\infty}$ is the space of (essentially) bounded functions of compact support. The central space is, of course, $L^{2}$.

An interesting subspace of the preceeding space is the LHS $V_{\gamma}$ generated by the weight functions $r_{\alpha}(x)=\exp \alpha x$, for $-\gamma \leqslant \alpha \leqslant \gamma(\gamma>0)$. Then all the spaces of the lattice may be obtained by interpolation from $L^{2}\left(r_{ \pm \gamma}\right)$, and moreover, the extreme spaces are themselves Hilbert spaces, namely

$$
\begin{aligned}
V_{\gamma}^{\#} & =L^{2}\left(\mathbb{R}, e^{-\gamma x} d x\right) \cap L^{2}\left(\mathbb{R}, e^{\gamma x} d x\right) \simeq L^{2}\left(\mathbb{R}, e^{-\gamma|x|} d x\right) \\
V_{\gamma} & =L^{2}\left(\mathbb{R}, e^{-\gamma x} d x\right)+L^{2}\left(\mathbb{R}, e^{\gamma x} d x\right) \simeq L^{2}\left(\mathbb{R}, e^{\gamma|x|} d x\right) .
\end{aligned}
$$

This LHS plays an interesting role in scattering theory [13].

Actually the whole construction goes through if one takes for $\mathcal{H}_{r}$ a reflexive Banach space, as in interpolation theory [22]. In this way one recovers the families $\left\{\ell^{p}\right\}$ or $\left\{L^{p}\right\}(1<$ $p<\infty)$ discussed in Section 4. For simplicity we restrict the discussion to a LHS.

Let $V_{I}=\left\{\mathcal{H}_{r}, r \in I\right\}$ be a LHS. The whole idea behind this structure (as for general PIP-spaces) is that vectors should not be considered individually, but only in terms of the subspaces $\mathcal{H}_{r}$, which are the building blocks of the theory. The same spirit determines the definition of an operator on a LHS space: only bounded operators between Hilbert spaces are allowed, but an operator is a (maximal) coherent collection of these. To be more specific, an operator on $V_{I}$ is a map $A: \mathcal{D}(A) \rightarrow V$, such that:

(i) $\mathcal{D}(A)=\bigcup_{q \in D(A)} \mathcal{H}_{q}$, where $D(A)$ is a nonempty subset of $I$. 
(ii) For every $q \in D(A)$, there is $p \in I$ such that the restriction $A: \mathcal{H}_{q} \rightarrow \mathcal{H}_{p}$ is linear and bounded (we denote it by $A_{p q} \in \mathcal{B}\left(\mathcal{H}_{q}, \mathcal{H}_{p}\right)$ ).

(iii) $A$ has no proper extension satisfying (i) and (ii).

The bounded linear operator $A_{p q}: \mathcal{H}_{q} \rightarrow \mathcal{H}_{p}$ is called a representative of $A$. Thus $A$ is characterized by two subsets of $I: 1$

$$
\begin{aligned}
D(A) & =\left\{q \in I: \text { there is a } p \text { such that } A_{p q} \text { exists }\right\} \\
I(A) & =\left\{p \in I: \text { there is a } q \text { such that } A_{p q} \text { exists }\right\}
\end{aligned}
$$

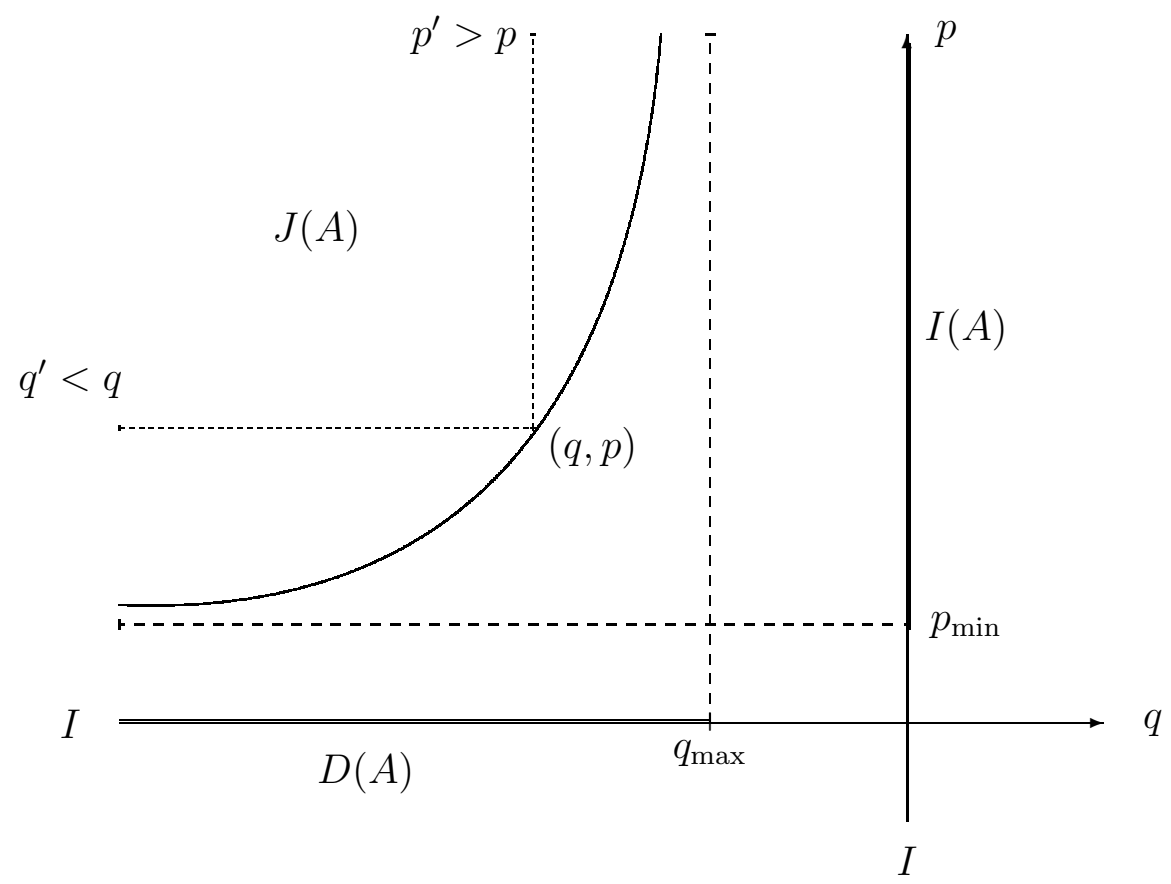

Figure 4 : The various sets characterizing the operator $A$ (in the case of a scale).

We denote by $J(A)$ the set of all such pairs $(q, p)$ for which $A_{p q}$ exists. Thus the operator $A$ is equivalent to the collection of its representatives

$$
A \simeq\left\{A_{p q}:(q, p) \in J(A)\right\}
$$

$D(A)$ is an initial subset of $I$ : if $q \in D(A)$ and $q^{\prime}<q$, then $q^{\prime} \in D(A)$, and $A_{p q^{\prime}}=A_{p q} E_{q q^{\prime}}$, where $E_{q q^{\prime}}$ is the unit operator (this is what we mean by 'coherent'). In the same way,

\footnotetext{
${ }^{1}$ The set $I(A)$ was denoted $R(A)$ in $[3]$, but this obviously conflicts with the space of right multipliers, to be defined below.
} 
$I(A)$ is a final subset of $I$ : if $p \in I(A)$ and $p^{\prime}>p$, then $p^{\prime} \in I(A)$. Figure 4 illustrates the situation in the case of a Hilbert scale ( $I$ totally ordered). Notice that, even then, the extreme elements $q_{\max }=\bigvee_{q \in D(A)} q$, resp. $p_{\min }=\bigwedge_{p \in I(A)} q$ need not belong to $D(A)$, resp. $I(A)$, since $I$ is not a complete lattice in general. Also $J(A) \subset D(A) \times I(A)$, with strict inclusion in general.

We denote by $O p\left(V_{I}\right)$ the set of all operators on $V_{I}$. Since $V^{\#} \subset \mathcal{H}_{r}, \forall r \in I$, an operator may be identified with a sesquilinear form on $V^{\#} \times V^{\#}$. Indeed, the restriction of any representative $A_{p q}$ to $V^{\#} \times V^{\#}$ is such a form, and they all coincide. Equivalently, an operator may be identified with a linear map from $V^{\#}$ into $V$. But the idea behind the notion of operator is to keep also the algebraic operations on operators, namely:

(i) Adjoint $A^{*}$ : every operator $A \in O p\left(V_{I}\right)$ has a unique adjoint $A^{*} \in O p\left(V_{I}\right)$, defined by:

$$
\left\langle A^{*} x \mid y\right\rangle=\langle x \mid A y\rangle, \text { for } y \in \mathcal{H}_{r}, r \in J(A) \text { and } x \in V_{\bar{s}}, s \in I(A),
$$

that is, $\left(A^{*}\right)_{\overline{r s}}=\left(A_{s r}\right)^{*}$ (usual Hilbert space adjoint). This implies that $A^{* *}=$ $A, \forall A \in O p\left(V_{I}\right)$ : no extension is allowed, because of the maximality condition (iii).

(ii) Partial multiplication : $A B$ is defined iff there is a $q \in I(B) \cap D(A)$, that is, iff there is continuous factorization through some $\mathcal{H}_{q}$ :

$$
\mathcal{H}_{r} \stackrel{B}{\rightarrow} \mathcal{H}_{q} \stackrel{A}{\rightarrow} \mathcal{H}_{s}, \quad \text { i.e. } \quad(A B)_{s r}=A_{s q} B_{q r} .
$$

Notice that here, contrary to the case of a general PIP-space, the domain $\mathcal{D}(A)$ is automatically a vector subspace of $V$. Therefore $O p\left(V_{I}\right)$ is a partial *-algebra (which means, in particular, that the usual rule of distributivity is valid).

Now we turn to the spaces of multipliers. Our building blocks are the sets:

$$
\mathcal{O}_{p q}=\left\{A \in O p\left(V_{I}\right): A_{p q} \text { exists }\right\}
$$

Clearly we have:

- $L \mathcal{O}_{p q} \equiv L_{p}=\left\{C \in O p\left(V_{I}\right): p \in D(C)\right\}=\bigcup_{s} \mathcal{B}\left(\mathcal{H}_{p}, \mathcal{H}_{s}\right) \simeq \operatorname{End}\left(\mathcal{H}_{p}, V\right)$

- $R \mathcal{O}_{p q} \equiv R_{q}=\left\{B \in O p\left(V_{I}\right): q \in I(B)\right\}=\bigcup_{t} \mathcal{B}\left(\mathcal{H}_{t}, \mathcal{H}_{q}\right) \simeq \operatorname{End}\left(V^{\#}, \mathcal{H}_{q}\right)$

- $R L \mathcal{O}_{p q}=R L_{p}=R_{p} \in \mathcal{F}^{R}$

- $L R \mathcal{O}_{p q}=L R_{q}=L_{p} \in \mathcal{F}^{L}$.

(in these relations, End $(X, Y)$ denotes the space of all linear maps from $X$ into $Y$ ). 
From this we deduce immediately, using the fact that $L, R$ are lattice anti-isomorphisms:

$$
\begin{array}{ll}
L_{p} \wedge L_{q}=L_{p \vee q} & L_{p} \vee L_{q}=L_{p \wedge q} \\
R_{p} \wedge R_{q}=R_{p \wedge q} & R_{p} \vee R_{q}=R_{p \vee q} .
\end{array}
$$

In particular, $q \leqslant q^{\prime}$ implies $R_{q} \subset R_{q^{\prime}}$ and $L_{q} \supset L_{q^{\prime}}$. Thus $\mathcal{I}^{L}=\left\{L_{p}\right\}$ is a sublattice of $\mathcal{F}^{L}, \mathcal{I}^{R}=\left\{R_{p}\right\}$ is a sublattice of $\mathcal{F}^{R}$, and both are generating (except that they do not contain the extreme elements in general, see below). In addition $\mathcal{I}^{L}, \mathcal{I}^{R}$ consist of matching pairs $\left(R_{q}, L_{q}\right)$. Indeed, given $A \in O p\left(V_{I}\right)$, we may rewrite

$$
D(A)=\left\{q \in I \mid A \in L_{q}\right\}, \quad I(A)=\left\{p \in I \mid A \in R_{p}\right\}
$$

and therefore

$$
A \in L(B) \quad \Leftrightarrow \quad \exists p \in I \text { such that } A \in L_{p}, B \in R_{p} .
$$

From (5.4), we deduce individual multiplier spaces:

$$
L(A)=\bigvee_{p \in I(A)} L_{p}=L_{p_{\min }}, \quad R(A)=\bigvee_{q \in D(A)} R_{q}=R_{q_{\max }}
$$

Note that these two subsets do not belong to $\mathcal{I}^{L}$, resp. $\mathcal{I}^{R}$, in general, but to the complete lattice generated by the latter.

In the same way, we obtain

$$
R O p\left(V_{I}\right)=\{A \mid I(A)=I\}=\bigcap_{r \in I} R_{r}
$$

which may be identified with the space End $\left(V^{\#}\right)$ of all linear maps from $V^{\#}$ into itself. Again, $R O p\left(V_{I}\right) \notin \mathcal{I}^{R}$. Similarly,

$$
\operatorname{LOp}\left(V_{I}\right)=\{A \mid D(A)=I\}=\bigvee_{p \in I} L_{p} \simeq \operatorname{End}(V)
$$

The final point concerns topologies on spaces of multipliers. As a consequence of the identification (5.1) of an operator with the set of its representatives, the partial *-algebra $O p\left(V_{I}\right)$ itself has the structure of an inductive limit of Banach spaces:

$$
O p\left(V_{I}\right) \simeq \bigcup_{q, p \in I} \mathcal{B}\left(\mathcal{H}_{q}, \mathcal{H}_{p}\right)
$$

One may also consider the extreme spaces $V^{\#}=\bigcap_{r \in I} \mathcal{H}_{r}, V=\sum_{r \in I} \mathcal{H}_{r}$. On $V$, the inductive limit topology coincides with the Mackey topology $\tau\left(V, V^{\#}\right)$, but on $V^{\#}$, the projective topology may be coarser than the Mackey topology $\tau\left(V^{\#}, V\right)$. This gives another possibility of giving a topology to $O p\left(V_{I}\right)$, by identifying an operator with a 
continuous linear map from $V^{\#}$ into $V$ (each of them endowed with its own Mackey topology), that is:

$$
O p\left(V_{I}\right) \simeq \mathcal{L}\left(V^{\#}, V\right) .
$$

These various possibilities may be different in general, which makes the problem quite involved. Instead we will consider several simpler cases.

(1) First, suppose that the extreme spaces $V^{\#}$ and $V$ are themselves Hilbert spaces, as for the LHS $V_{\gamma}$ described above, or, in the Banach case, the lattices $\left\{\ell^{p}\right\},\left\{L^{p}[0,1]\right\}$, $\left\{L^{p}(\mathbb{R})\right\},\left\{\left(L^{p}, \ell^{q}\right)\right\}$. In that case, the relation (5.9) gives immediately the identification $O p\left(V_{I}\right) \simeq \mathcal{B}\left(V^{\#}, V\right)$, with its usual norm topology. Similarly, $R O p\left(V_{I}\right) \simeq \mathcal{B}\left(V^{\#}\right)$ and $L O p\left(V_{I}\right) \simeq \mathcal{B}(V)$. More generally

$$
\begin{aligned}
L_{q} & =\text { ind } \lim _{t} \mathcal{B}\left(\mathcal{H}_{q}, \mathcal{H}_{t}\right) \simeq \mathcal{B}\left(\mathcal{H}_{q}, V\right), \\
R_{q} & =\text { ind } \lim _{s} \mathcal{B}\left(\mathcal{H}_{s}, \mathcal{H}_{q}\right) \simeq \mathcal{B}\left(V^{\#}, \mathcal{H}_{q}\right),
\end{aligned}
$$

and these norm topologies coincide with the topologies $\lambda$, resp. $\rho$, on $L_{q}$, resp. $R_{q}$. Finally the involution is clearly continuous on $O p\left(V_{I}\right)$, so that $O p\left(V_{I}\right)$ is a topological partial *-algebra. However, tightness is open in general.

(2) The situation is still simple, and most of the results of (1) survive, when $V_{I}$ consists of a scale (either continuous or discrete) of Hilbert spaces. Then, indeed, $I$ contains a countable subset $J$, stable under the involution, and coinitial to $I$, which means that, for each $r \in I$, there exists $q \in J$ such that $q \leqslant r$ ( $J$ is then automatically cofinal to $I$ : $\forall r \in I$, there exists $p \in J$ such that $r \leqslant p$ ). As a consequence, the projective topology $t_{I}$, defined by $V_{I}$, is equivalent to that defined by $V_{J}=\left\{\mathcal{H}_{s}, s \in J\right\}$. In this case

$$
V^{\#}=\bigcap_{s \in J} \mathcal{H}_{s}, \quad V=\bigcup_{s \in J} \mathcal{H}_{s}
$$

and hence $V^{\#}$ is a reflexive Fréchet space and $V$ is a reflexive DF-space. Thus the projective topology on $V^{\#}$ coincides with the Mackey topology $\tau\left(V^{\#}, V\right)$, and no pathology arises. The space $\mathcal{L}\left(V^{\#}, V\right)$ of Mackey continuous operators coincides exactly with the space of all linear maps from $V^{\#}$ into $V$ which are continuous from $V^{\#}\left[t_{I}\right]$ into $V\left[t_{I}^{\prime}\right]$, where $\left[t_{I}^{\prime}\right]$ denotes the strong dual topology. In this situation, the space $\mathcal{L}\left(V^{\#}, V\right)$ provides an example of a quasi*-algebra of operators [29, 37] and the usual theory applies.

In particular, in addition to (5.9), we have the identifications:

$$
R O p\left(V_{I}\right) \simeq \mathcal{L}\left(V^{\#}\right), \quad L O p\left(V_{I}\right) \simeq \mathcal{L}(V),
$$

where $\mathcal{L}\left(V^{\#}\right)$ is the space of all continuous operators from $V^{\#}\left[t_{I}\right]$ into itself and $\mathcal{L}(V)$ and the space of all continuous operators from $V\left[t_{I}^{\prime}\right]$ into itself (both these spaces can be 
identified with subspaces of $\left.\mathcal{L}\left(V^{\#}, V\right)\right)$. Similarly one gets:

$$
L_{q} \simeq \mathcal{L}\left(\mathcal{H}_{q}, V\right), \quad R_{q} \simeq \mathcal{L}\left(V^{\#}, \mathcal{H}_{q}\right), \quad q \in I
$$

Of course, these results remain valid if $I$ is not a scale, but a lattice containing a countable subset $J=\bar{J}$, coinitial to $I: V^{\#}$ is Fréchet and $V$ a DF-space.

Topologies on $\mathcal{L}\left(V^{\#}, V\right)$ can then be introduced following [29]. The most interesting seems to be the uniform topology defined by the set of seminorms

$$
A \in \mathcal{L}\left(V^{\#}, V\right) \mapsto \sup _{f, g \in \mathcal{M}}|\langle f \mid A g\rangle|
$$

where $\mathcal{M}$ is a bounded subset of $V^{\#}\left[t_{I}\right]$. Then there are several possible ways of turning $\mathcal{L}\left(V^{\#}, V\right)$ into a partial *-algebra, in such a way that one always has, as in (5.11):

$$
R \mathcal{L}\left(V^{\#}, V\right)=\mathcal{L}\left(V^{\#}\right), \quad L \mathcal{L}\left(V^{\#}, V\right)=\mathcal{L}(V)
$$

Since the involution and the multiplications are continuous with respect to the uniform topology, $\mathcal{L}\left(V^{\#}, V\right)$ becomes a topological partial *-algebra, no matter how many Hilbert spaces we use to define (by composition) the multiplication (provided that the relations (5.13) are satisfied). The simplest possibility, usually adopted in the theory of quasi * algebras, consists in considering none of them: this choice yields very poor lattices of multipliers (for instance $\mathcal{J}^{R}$ contains only $R \mathcal{L}\left(V^{\#}, V\right)$ and $\mathcal{L}\left(V^{\#}, V\right)$ itself). With this trivial lattice of multipliers, $\mathcal{L}\left(V^{\#}, V\right)$ is a tight topological partial *-algebra for wellbehaved spaces $V^{\#}$, typically a Fréchet space whose topology is the projective topology generated by an $\mathrm{O}^{*}$-algebra. In that case indeed, both $\mathcal{L}\left(V^{\#}\right)$ and $\mathcal{L}(V)$ are uniformly dense in $\mathcal{L}\left(V^{\#}, V\right)$ [28, 34].

But this was clearly not what we had in mind when we considered a LHS! We were, in fact interested in finding a larger (and possibly the largest) lattice of multipliers, making use of the factorization via the spaces $\left\{\mathcal{H}_{p}, p \in I\right\}$ (this corresponds, of course, to the possiblility of getting the largest possible set of multiplicable pairs). As said before, in all these cases, $\mathcal{L}\left(V^{\#}, V\right)$ is a topological partial *-algebra, but tightness is still to be proven. It is interesting to notice the analogy of this procedure of 'enrichment' of the lattice of multiplier spaces with the similar operation of refinement or coarsening of a compatibility relation, which also leads to the construction of suitable lattices of subspaces, either containing, or contained in, the corresponding lattice as a sublattice (see [2, 5] for a systematic discussion). One should also beware of possible pathologies linked to associativity, discovered by Kürsten [27].

One of the most interesting cases for applications is that of the Hilbert scale built on the powers of a self-adjoint operator $H>1$. That is, $\mathcal{I}=\left\{\mathcal{H}_{s}, s \in I \equiv \mathbb{R}\right.$ or $\left.\mathbb{Z}\right\}$, where $\mathcal{H}_{s}=D\left(H^{s}\right), s \geqslant 0$, with the graph norm, and $H_{-s}=\mathcal{H}_{s}^{\times}, V^{\#}=\bigcap_{s \in I} \mathcal{H}_{s}=$ 
$D^{\infty}(H), V=\sum_{s \in I} \mathcal{H}_{s}$. The partial multiplication in $O p\left(V_{I}\right) \simeq \mathcal{L}\left(V^{\#}, V\right)$ is defined by continuous factorization through some $\mathcal{H}_{s}: A \cdot B$ is defined whenever there exists $s \in I$ such that $B \in \mathcal{L}\left(V^{\#}, \mathcal{H}_{s}\right)$ and $A \in \mathcal{L}\left(\mathcal{H}_{s}, V\right)$. The spaces of multipliers themselves, given in (5.12), form scales:

$$
\mathcal{I}^{L}=\left\{L_{s}=\mathcal{L}\left(\mathcal{H}_{s}, V\right), s \in I\right\}, \quad \mathcal{I}^{R}=\left\{R_{s}=\mathcal{L}\left(V^{\#}, \mathcal{H}_{s}\right), s \in I\right\}
$$

In the case of a discrete scale, $I=\mathbb{Z}$, the lattices $\mathcal{I}^{L}, \mathcal{I}^{R}$ are already complete. For instance, if $K$ is a subset of $\mathbb{Z}$, bounded from above, then $\bigcap_{n \in K} R_{n}=R_{n_{K}}$, with $n_{K}=$ $\max K$. For a continuous scale, $I=\mathbb{R}$, this is no longer the case, but the lattice completion is obtained exactly as in the case of the $L^{p}$ spaces described in Section 4 , by 'enriching' the line $\mathbb{R}$. For instance,

$$
\mathcal{H}_{s-}=\bigcap_{r<s} \mathcal{H}_{r}, \quad \mathcal{H}_{s+}=\bigcup_{t>s} \mathcal{H}_{t}
$$

With their projective, resp. inductive topology, $\mathcal{H}_{s-}$ is a reflexive Fréchet space and $\mathcal{H}_{s+}$ is a reflexive DF-space. The rest is as before, duality relations and lattice completions. The interesting point is the following result.

Proposition 5.1 - Let $\mathcal{I}=\left\{\mathcal{H}_{s}, s \in I \equiv \mathbb{R}\right.$ or $\left.\mathbb{Z}\right\}$ be the Hilbert scale built on the powers of a self-adjoint operator $H>1$, with $V^{\#}=D^{\infty}(H), V=\sum_{s \in I} \mathcal{H}_{s}$. Then, with partial multiplication defined by continuous factorization through the spaces $\mathcal{H}_{s}, O p\left(V_{I}\right) \simeq$ $\mathcal{L}\left(V^{\#}, V\right)$ is a tight topological partial *-algebra with respect to the uniform topology.

The proof is given in the Appendix. Here instead, let us consider the two examples already mentioned:

(i) The Hilbert scale around $L^{2}(\mathbb{R}, d x)$ built on the powers of the self-adjoint operator $H=\frac{1}{2}\left(-\frac{d^{2}}{d x^{2}}+x^{2}\right)$ (this is the Hamiltonian of a quantum mechanical harmonic oscillator in one dimension). Going to the limits $n \rightarrow \pm \infty$ yields

$$
V^{\#}=\bigcap_{n \in \mathbb{Z}} \mathcal{H}_{n}=\mathcal{S}(\mathbb{R}) \quad \text { and } \quad V=\sum_{n \in \mathbb{Z}} \mathcal{H}_{n}=\mathcal{S}^{\prime}(\mathbb{R}),
$$

Schwartz' spaces of smooth fast decreasing functions and tempered distributions, respectively. In fact, this scale may be used for a simpler formulation of the theory of tempered distributions, called the Hermite representation [35]. This example illustrate the usefulness of considering $O p\left(V_{I}\right) \simeq \mathcal{L}\left(\mathcal{S}, \mathcal{S}^{\prime}\right)$ as a partial *-algebra. As a consequence of Proposition 5.1, the space $R O p\left(V_{I}\right)=\mathcal{L}(\mathcal{S})$ is dense in every $R_{n}=\mathcal{L}\left(\mathcal{S}, \mathcal{H}_{n}\right)$. Thus we have a tight topological partial *-algebra, already studied in 32 . 
(ii) The Sobolev scale $\left\{W_{s}^{2}\left(\mathbb{R}^{n}\right), s \in \mathbb{R}\right\}$ is also of this type, with $H=1-\Delta$, acting in $L^{2}\left(\mathbb{R}^{n}, d^{n} x\right)$ ( $\Delta$ is the $n$-dimensional Laplacian). The operators on this scale are the building blocks of the theory of partial differential operators. Again the point of view of a topological partial *-algebra might be useful in applications, in particular the tightness condition may offer useful approximations. Notice that, if we take together the scale $\left\{W_{s}^{2}\right\}$ and its Fourier transform $\left\{\widehat{W_{s}^{2}}\right\}$, we recover the Schwartz spaces $\mathcal{S}, \mathcal{S}^{\prime}$ as extreme spaces.

In the general case, where $I$ does not contain a countable coinitial subset (or sublattice) $J$, things get quite involved. Standard examples are the full LHS of weighted $\ell^{2}$ or $L^{2}$ spaces described above. It is probably pointless to treat the problem in such generality.

\subsection{Partial $\mathrm{O}^{*}$-algebras}

Let $\mathcal{H}$ be a complex Hilbert space with inner product $\langle\cdot \mid \cdot\rangle$ and $\mathcal{D}$ a dense subspace of $\mathcal{H}$. We denote by $\mathcal{L}^{\dagger}(\mathcal{D}, \mathcal{H})$ the set of all (closable) linear operators $X$ such that $\mathcal{D}(X)=\mathcal{D}, \mathcal{D}\left(X^{*}\right) \supseteq \mathcal{D}$. The set $\mathcal{L}^{\dagger}(\mathcal{D}, \mathcal{H})$ is a partial *-algebra with respect to the following operations : the usual sum $X_{1}+X_{2}$, the scalar multiplication $\lambda X$, the involution $X \mapsto X^{\dagger}=X^{*}\left\lceil\mathcal{D}\right.$ and the (weak) partial multiplication $X_{1} \square X_{2}=X_{1}{ }^{\dagger *} X_{2}$, defined whenever $X_{2}$ is a weak right multiplier of $X_{1}, X_{2} \in R^{\mathrm{w}}\left(X_{1}\right)$, that is, iff $X_{2} \mathcal{D} \subset \mathcal{D}\left(X_{1}^{\dagger *}\right)$ and $X_{1}{ }^{*} \mathcal{D} \subset \mathcal{D}\left(X_{2}{ }^{*}\right)$. It is easy to check that $X_{1} \square X_{2}$ is well-defined iff there exists $C \in \mathcal{L}^{\dagger}(\mathcal{D}, \mathcal{H})$ such that

$$
\left\langle X_{2} f \mid X_{1}^{\dagger} g\right\rangle=\langle C f \mid g\rangle, \quad \forall f, g \in \mathcal{D} ;
$$

in this case $X_{1} \square X_{2}=C$. When we regard $\mathcal{L}^{\dagger}(\mathcal{D}, \mathcal{H})$ as a partial *-algebra with those operations, we denote it by $\mathcal{L}_{\mathrm{w}}^{\dagger}(\mathcal{D}, \mathcal{H})$.

A partial $\mathrm{O}^{*}$-algebra on $\mathcal{D}$ is a ${ }^{*}$-subalgebra $\mathfrak{M}$ of $\mathcal{L}_{\mathrm{w}}^{\dagger}(\mathcal{D}, \mathcal{H})$, that is, $\mathfrak{M}$ is a subspace of $\mathcal{L}_{\mathrm{w}}^{\dagger}(\mathcal{D}, \mathcal{H})$, containing the identity and such that $X^{\dagger} \in \mathfrak{M}$ whenever $X \in \mathfrak{M}$ and $X_{1} \square X_{2} \in \mathfrak{M}$ for any $X_{1}, X_{2} \in \mathfrak{M}$ such that $X_{2} \in R^{\mathrm{w}}\left(X_{1}\right)$. As for $\mathcal{L}_{\mathrm{w}}^{\dagger}(\mathcal{D}, \mathcal{H})$ itself, it is the largest partial $\mathrm{O}^{*}$-algebra on the domain $\mathcal{D}$. The sets $R \mathcal{L}_{\mathrm{w}}^{\dagger}(\mathcal{D}, \mathcal{H})$ of the universal right multipliers of $\mathcal{L}_{\mathrm{w}}^{\dagger}(\mathcal{D}, \mathcal{H})$ and $L \mathcal{L}_{\mathrm{w}}^{\dagger}(\mathcal{D}, \mathcal{H})$ of the universal left multipliers of $\mathcal{L}_{\mathrm{w}}^{\dagger}(\mathcal{D}, \mathcal{H})$ can be described as follows [6]:

$$
R \mathcal{L}_{\mathrm{w}}^{\dagger}(\mathcal{D}, \mathcal{H})=\left\{B \in \mathcal{L}^{\dagger}(\mathcal{D}, \mathcal{H}): \bar{B} \in \mathcal{B}(\mathcal{H}), B \mathcal{D} \subset \mathcal{D}^{*}\right\},
$$

where

$$
\mathcal{D}^{*}=\bigcap_{A \in \mathcal{L}^{\dagger}(\mathcal{D}, \mathcal{H})} D\left(A^{*}\right)
$$

and

$$
L \mathcal{L}_{\mathrm{w}}^{\dagger}(\mathcal{D}, \mathcal{H})=\left\{B^{*}: B \in R \mathcal{L}_{\mathrm{w}}^{\dagger}(\mathcal{D}, \mathcal{H})\right\}
$$


(If $\mathcal{D}=\mathcal{D}^{*}, \mathcal{L}_{\mathrm{w}}^{\dagger}(\mathcal{D}, \mathcal{H})$ is said to be self-adjoint).

In order to introduce a topology on $\mathcal{L}_{\mathrm{w}}^{\dagger}(\mathcal{D}, \mathcal{H})$ it is convenient to endow $\mathcal{D}$ with a topology which makes each $A \in \mathcal{L}_{\mathrm{w}}^{\dagger}(\mathcal{D}, \mathcal{H})$ continuous. This can be done by defining the topology on $\mathcal{D}$ by the following family of seminorms

$$
f \mapsto\|A f\|, \quad A \in \mathcal{L}_{\mathrm{w}}^{\dagger}(\mathcal{D}, \mathcal{H})
$$

This topology will be denoted in what follows by $t_{\mathcal{L}^{\dagger}}$. Clearly, $t_{\mathcal{L}^{\dagger}}$ is the projective topology defined on $\mathcal{D}$ by $\mathcal{L}^{\dagger}(\mathcal{D}, \mathcal{H})$ and for this reason each $A \in \mathcal{L}_{\mathrm{w}}^{\dagger}(\mathcal{D}, \mathcal{H})$ is continuous from $\mathcal{D}$ into $\mathcal{H}$.

We will now define three topologies on $\mathcal{L}_{\mathrm{w}}^{\dagger}(\mathcal{D}, \mathcal{H})$ and check whether $\mathcal{L}_{\mathrm{w}}^{\dagger}(\mathcal{D}, \mathcal{H})$ is a topological partial *-algebra with respect to them.

Quasi-uniform topology, $\tau_{*}$

It is defined by the set of seminorms

$$
A \mapsto \sup _{f \in \mathcal{N}}\left(\|A f\|+\left\|A^{\dagger} f\right\|\right), \quad \mathcal{N} \text { bounded in } \mathcal{D}\left[t_{\mathcal{L}^{\dagger}}\right]
$$

By the definition itself of $\tau_{*}$ it follows that the map $A \mapsto A^{\dagger}$ is continuous.

If $\mathfrak{M} \in \mathcal{F}^{R}$, then the corresponding topology $\rho_{\mathfrak{M}}^{*}$ is defined by the set of seminorms

$$
A \in \mathfrak{M} \mapsto \sup _{f \in \mathcal{N}}\left(\|(X \square A) f\|+\left\|\left(A^{\dagger} \square X^{\dagger}\right) f\right\|\right), \quad X \in L \mathfrak{M}, \mathcal{N} \text { bounded in } \mathcal{D}\left[t_{\mathcal{L}^{\dagger}}\right]
$$

The following lemma, proved in [6, 7], shows that if $\mathcal{L}_{\mathrm{w}}^{\dagger}(\mathcal{D}, \mathcal{H})$ is self-adjoint, the first two conditions of Definition 3.5 are fulfilled if $\mathcal{L}_{\mathrm{w}}^{\dagger}(\mathcal{D}, \mathcal{H})$ is endowed with $\tau_{*}$.

Lemma 5.2 - If $\mathcal{L}_{\mathrm{w}}^{\dagger}(\mathcal{D}, \mathcal{H})$ is self-adjoint, then the maps $A \mapsto X \square A$ and $A \mapsto A \square Y$ are $\tau_{*}$-continuous for all $X \in L \mathcal{L}_{\mathrm{w}}^{\dagger}(\mathcal{D}, \mathcal{H})$ and $Y \in R \mathcal{L}_{\mathrm{w}}^{\dagger}(\mathcal{D}, \mathcal{H})$; thus $\mathcal{L}_{\mathrm{w}}^{\dagger}(\mathcal{D}, \mathcal{H})\left[\tau_{*}\right]$ is a topological partial *-algebra.

The topology on $\mathfrak{M} \in \mathcal{F}^{R}$, defined as in Section 3 , will be called here $\rho_{\mathfrak{M}}^{*}$ to remind its dependence on $\tau_{*}$. Analogously, if $\mathfrak{N} \in \mathcal{F}^{L}$, its topology will be called $\lambda_{\mathfrak{N}}^{*}$.

By Lemma 3.1 and Lemma 5.2 it follows that the topologies $\rho_{\mathcal{L}_{\mathrm{w}}^{\dagger}(\mathcal{D}, \mathcal{H})}$ and $\lambda_{\mathcal{L}_{\mathrm{w}}^{\dagger}(\mathcal{D}, \mathcal{H})}$ both coincide with $\tau_{*}$.

The following result, in a slight different form, has been proved in [6]:

Proposition $5.3-\mathcal{L}_{\mathrm{w}}^{\dagger}(\mathcal{D}, \mathcal{H})$ is complete in $\tau_{*}$. If $\mathfrak{M} \in \mathcal{F}^{R}$, then $\mathfrak{M}$ is complete in $\rho_{\mathfrak{M}}^{*}$. Similarly, if $\mathfrak{N} \in \mathcal{F}^{L}$, then $\mathfrak{N}$ is complete in $\lambda_{\mathfrak{N}}^{*}$.

As for the third condition of Definition [3.5, the question as to whether $R \mathcal{L}_{\mathrm{w}}^{\dagger}(\mathcal{D}, \mathcal{H})$ is $\rho_{\mathfrak{M}}^{*}$-dense in each $\mathfrak{M} \in \mathcal{F}^{R}$ (i.e. the tightness of $\mathcal{L}_{\mathrm{w}}^{\dagger}(\mathcal{D}, \mathcal{H})\left[\tau_{*}\right]$ ) is still open. 
Strong* topology, $\tau_{\mathrm{s}} *$

With an obvious generalization of the case of bounded operator algebras, the strong* topology on $\mathcal{L}^{\dagger}(\mathcal{D}, \mathcal{H})$ is defined by the set of seminorms

$$
A \mapsto\|A f\|+\left\|A^{\dagger} f\right\|, \quad f \in \mathcal{D} .
$$

This topology plays a fundamental role in the study of unbounded commutants [12]. The map $A \mapsto A^{\dagger}$ is continuous by definition, but as in the case of $\mathcal{B}(\mathcal{H})$, the multiplications may fail to be continuous. Therefore $\mathcal{L}_{\mathrm{w}}^{\dagger}(\mathcal{D}, \mathcal{H})\left[\tau_{\mathrm{s}} *\right]$ is not a topological partial *-algebra. Nevertheless, the close connection between the strong* topology and commutants allows to get some interesting density theorem. We will show, in fact, that the set

$$
\mathcal{B}=\left\{B \in \mathcal{L}^{\dagger}(\mathcal{D}, \mathcal{H}): \bar{B} \in \mathcal{B}(\mathcal{H}) ; B \mathcal{D} \subseteq \mathcal{D}\right\}
$$

is dense in $\mathcal{L}_{\mathrm{w}}^{\dagger}(\mathcal{D}, \mathcal{H})\left[\tau_{\mathrm{s}} *\right]$. This result is a consequence of the following stronger statement.

Proposition 5.4 - The *-algebra $\mathcal{F}$ generated by the the identity operator and by the set $\mathcal{F}(\mathcal{D})$ of all finite rank operators in $\mathcal{D}$ is dense in $\mathcal{L}_{\mathrm{w}}^{\dagger}(\mathcal{D}, \mathcal{H})\left[\tau_{\mathrm{s}} *\right]$

Proof. - By [31, Proposition 9], the $\tau_{\mathrm{s}}$-closure of $\mathcal{F}$ is $\mathcal{F}_{\sigma \sigma}^{\prime \prime}$, the weak unbounded bicommutant of $\mathcal{F}$. For this reason it is enough to prove that $\mathcal{F}_{\sigma}^{\prime}$ consists only of multiples of the identity operator. Now, let $X \in \mathcal{F}_{\sigma}^{\prime}$; then $X$ commutes (weakly) with each $P_{\phi}$, $\phi \in \mathcal{D}$ where $P_{\phi} \psi=(\phi, \psi) \phi$. Therefore,

$$
X \phi=\frac{1}{\|\phi\|^{2}} X P_{\phi} \phi=\frac{1}{\|\phi\|^{2}} P_{\phi} X \phi=\frac{(\phi, X \phi)}{\|\phi\|^{2}} \phi .
$$

Now starting from two elements $\phi_{1}, \phi_{2} \in \mathcal{D}$ such that $\left(\phi_{1}, \phi_{2}\right)=0$ and using the linearity, it is easy to show that the coefficient $\frac{(\phi, X \phi)}{\|\phi\|^{2}}$ does not depend on $\phi$.

Weak topology, $\tau_{\mathrm{w}}$

It is defined by the set of seminorms

$$
A \mapsto|\langle f \mid A g\rangle|, \quad f, g \in \mathcal{D}
$$

Also in this case it is readily checked that the map $A \mapsto A^{\dagger}$ is continuous.

If $\mathfrak{M} \in \mathcal{F}^{R}$, then the corresponding topology $\rho_{\mathfrak{M}}^{\mathrm{w}}$ is defined by the set of seminorms

$$
A \in \mathfrak{M} \rightarrow|\langle f \mid(X \square A) g\rangle|, \quad X \in L \mathfrak{M}, f, g \in \mathcal{D} \text {. }
$$

It is very easy to prove the following

Lemma 5.5 - If $\mathcal{L}_{\mathrm{w}}^{\dagger}(\mathcal{D}, \mathcal{H})$ is self-adjoint, then the maps $A \rightarrow X \square A$ and $A \rightarrow A \square Y$ are $\tau_{\mathrm{w}}$-continuous for all $X \in L \mathcal{L}_{\mathrm{w}}^{\dagger}(\mathcal{D}, \mathcal{H})$ and $Y \in R \mathcal{L}_{\mathrm{w}}^{\dagger}(\mathcal{D}, \mathcal{H})$. 
The previous lemma shows that $\mathcal{L}_{\mathrm{w}}^{\dagger}(\mathcal{D}, \mathcal{H})\left[\tau_{\mathrm{w}}\right]$ is a topological partial *-algebra, at least when $\mathcal{L}_{\mathrm{w}}^{\dagger}(\mathcal{D}, \mathcal{H})$ is self-adjoint. We will now consider the density condition of Definition 3.5. In order to get results in this direction it is useful to have at hand some information on the $\tau_{\mathrm{w}}$-continuous functionals on $\mathcal{L}_{\mathrm{w}}^{\dagger}(\mathcal{D}, \mathcal{H})$. In the very same way as in the case of weakly continuous functionals of $\mathcal{B}(\mathcal{H}$ ) (see e.g. [36, Ch. I]), we can prove the following:

Proposition 5.6 - For each $\tau_{\mathrm{w}}$-continuous linear functional $F$ on $\mathcal{L}_{\mathrm{w}}^{\dagger}(\mathcal{D}, \mathcal{H})$ there exist elements $f_{1}, \ldots, f_{n} ; g_{1}, \ldots, g_{n}$ in $\mathcal{D}$ such that

$$
F(X)=\sum_{i=1}^{n}\left\langle f_{i} \mid X g_{i}\right\rangle, \quad X \in \mathcal{L}_{\mathrm{w}}^{\dagger}(\mathcal{D}, \mathcal{H}) .
$$

Furthermore the vectors $f_{1}, \ldots, f_{n} ; g_{1}, \ldots, g_{n}$ can be chosen so that $\left\langle f_{i} \mid f_{j}\right\rangle=\delta_{i j}\left\|f_{i}\right\|^{2}$ and $\left\langle g_{i} \mid g_{j}\right\rangle=\delta_{i j}\left\|g_{i}\right\|^{2}$.

Making use of this result and of Lemma 3.6, we get easily that

Proposition 5.7 - Let $\mathfrak{M} \in \mathcal{F}^{R}$. Then, for each $\rho_{\mathfrak{M}}^{\mathrm{w}}$-continuous linear functional $F$ on $\mathfrak{M}$ there exist elements $f_{1}, \ldots, f_{n} ; g_{1}, \ldots, g_{n}$ in $\mathcal{D}$ and operators $A_{1}, \ldots A_{n}$ in $L \mathfrak{M}$ such that

$$
\left.\left.F(X)=\sum_{i=1}^{n}\left\langle f_{i}\right| A_{i} \square X\right) g_{i}\right\rangle, \quad X \in \mathfrak{M} .
$$

We now prove the following

Proposition $5.8-R \mathcal{L}_{\mathrm{w}}^{\dagger}(\mathcal{D}, \mathcal{H})$ is $\tau_{\mathrm{w}}$-dense in $\mathcal{L}_{\mathrm{w}}^{\dagger}(\mathcal{D}, \mathcal{H})$.

Proof. - Were it not so, there would exist a non-zero $\tau_{\mathrm{w}}$-continuous linear functional $F$ on $\mathcal{L}_{\mathrm{w}}^{\dagger}(\mathcal{D}, \mathcal{H})$ which is zero all over $R \mathcal{L}_{\mathrm{w}}^{\dagger}(\mathcal{D}, \mathcal{H})$. By Proposition 5.6, there exist elements $f_{1}, \ldots, f_{n} ; g_{1}, \ldots, g_{n}$ in $\mathcal{D}$ such that

$$
F(X)=\sum_{i=1}^{n}\left\langle f_{i} \mid X g_{i}\right\rangle, \quad X \in \mathcal{L}_{\mathrm{w}}^{\dagger}(\mathcal{D}, \mathcal{H})
$$

We choose the vectors $f_{1}, \ldots, f_{n} ; g_{1}, \ldots, g_{n}$ so that $\left\langle f_{i} \mid f_{j}\right\rangle=\delta_{i j}\left\|f_{i}\right\|^{2}$ and $\left\langle g_{i} \mid g_{j}\right\rangle=$ $\delta_{i j}\left\|g_{i}\right\|^{2}$.

The finite rank operator $X$ defined by

$$
X \varphi=\sum_{j=1}^{n}\left\langle f_{j} \mid \varphi\right\rangle g_{j}, \quad \varphi \in \mathcal{D}
$$

clearly belongs to $R \mathcal{L}_{\mathrm{w}}^{\dagger}(\mathcal{D}, \mathcal{H})$. Then we have

$$
F(X)=\sum_{i=1}^{n}\left\langle g_{i} \mid \sum_{i=1}^{n}\left\langle f_{i} \mid f_{j}\right\rangle g_{j}\right\rangle=\sum_{i=1}^{n}\left\|f_{i}\right\|^{2}\left\|g_{i}\right\|^{2}=0 .
$$


This implies $f_{1}=\ldots f_{n}=g_{1}=\ldots g_{n}=0$. Therefore $F=0$ and this contradicts the assumption.

Unfortunately, the argument used in this proof cannot be adapted to show that $R \mathcal{L}_{\mathrm{w}}^{\dagger}(\mathcal{D}, \mathcal{H})$ is $\rho_{\mathfrak{M}}^{\mathrm{w}}$-dense in each $\mathfrak{M} \in \mathcal{F}^{R}$, so that the tightness of this topological partial *-algebra remains to be proven.

So far we have considered only the largest partial $\mathrm{O}^{*}$-algebra, $\mathcal{L}_{\mathrm{w}}^{\dagger}(\mathcal{D}, \mathcal{H})$ itself. What about smaller ones? In view of the results of Section 4, one might hope that abelian partial O*-algebras would be topological partial *-algebras, possibly even tight ones. However, this is not the case, as shown by the following counterexample.

Let $T$ be a maximal symmetric operator and $\mathcal{D}=D\left(T^{n}\right), n<\infty$. Then [9, 11] the partial $\mathrm{O}^{*}$-algebra generated by $T^{[1]}=T\left\lceil\mathcal{D}\right.$ is the set $\mathfrak{P}_{n}\left(T^{[1]}\right)$ of polynomials of degree at most $n$, powers being defined as $T^{[n]}=T^{[1]} \square T^{[1]} \ldots \square T^{[1]}$. This is an abelian, finite dimensional partial $\mathrm{O}^{*}$-algebra. The partial multiplication is the usual weak multiplication and $P_{1} \square P_{2}$ is well-defined iff $\operatorname{deg}\left(P_{1}\right)+\operatorname{deg}\left(P_{2}\right) \leqslant n$. Thus, if $P_{j}$ has degree $j$, $M\left(P_{j}\right)=\mathfrak{P}_{n-j}\left(T^{[1]}\right)$, so that the set of multiplier spaces is the finite scale

$$
\mathfrak{P}_{0} \subset \mathfrak{P}_{1} \subset \ldots \subset \mathfrak{P}_{n}, \quad \mathfrak{P}_{j} \simeq \mathbb{C}^{j+1}
$$

In particular, $R \mathfrak{P}_{n}=\mathfrak{P}_{0}=\mathbb{C}$, which of course cannot be dense in any $\mathfrak{P}_{j}$. Thus $\mathfrak{P}_{n}\left(T^{[1]}\right)$ is a (trivial) nontight topological partial *-algebra. Additional exemples of the same nature may be found in [11].

For a general partial $\mathrm{O}^{*}$-algebra $\mathfrak{M}$, with partial multiplication $\square$, things are easy on the algebraic side. The multipliers to be used are, of course, the internal ones, such as $R \mathfrak{M}=R(\mathfrak{M}) \cap \mathfrak{M}$, and the whole lattice structure is the same as usual. However, the problem of topologies is quite difficult. It is already a nontrivial problem to find the spaces of multipliers explicitly, not to speak of proving that $\mathfrak{M}$ is a topological partial *-algebra! And the case of partial $\mathrm{GW}^{*}$-algebras does not seem simpler.

Remark. - Once we have endowed $\mathcal{D}$ with the topology $t_{\mathcal{L}^{\dagger}}$, it is natural to consider $\mathcal{L}^{\dagger}(\mathcal{D}, \mathcal{H})$ as a subspace of $\mathcal{L}\left(\mathcal{D}, \mathcal{D}^{\prime}\right)$ where $\mathcal{D}^{\prime}$ is the conjugate dual of $\mathcal{D}$ endowed with the strong dual topology $t_{\mathcal{L}^{\dagger}}^{\prime}$. In this case if $A, B \in \mathcal{L}^{\dagger}(\mathcal{D}, \mathcal{H})$ then the product $A \cdot B$ always exists in $\mathcal{L}\left(\mathcal{D}, \mathcal{D}^{\prime}\right)$. Indeed, each $A \in \mathcal{L}^{\dagger}(\mathcal{D}, \mathcal{H})$ has an extension $\widehat{A}$ (the transposed map of $\left.A^{\dagger}\right)$ which is continuous from $\mathcal{H}$ into $\mathcal{D}^{\prime}$. Then $A \cdot B$ is defined by $A \cdot B f=\widehat{A}(B f), f \in \mathcal{D}$. The definition of the multiplication - comes directly from the duality. This fact, together with Eq. (5.15) shows that if $A \square B$ is also well-defined, then necessarily $A \square B=A \cdot B$. (This is reminiscent of the notion of weak derivative in $L^{2}$ : given $f \in L^{2}$, its derivative exists always as a tempered distribution $f^{\prime} \in \mathcal{S}^{\prime}$, but $f$ belongs to the (Hilbert space) domain of $d / d x$ only if $\left.f^{\prime} \in L^{2}\right)$. 
One can go one step further if $\mathcal{D}=D^{\infty}(H)$, for some self-adjoint operator $H>1$. Then one may interpolate between $\mathcal{D}$ and $\mathcal{D}^{\prime}$ by the Hilbert scale $\left\{\mathcal{H}_{n}, n \in \mathbb{Z}\right\}$, as discuused in Section 5.1. The result is the same, , the partial multiplication on $\mathcal{L}\left(\mathcal{D}, \mathcal{D}^{\prime}\right)$ defined by continuous factorization through the spaces $\mathcal{H}_{n}$ coincides again with the weak partial multiplication $\square$.

\section{Outcome}

The definition of topological partial *-algebra that emerges from this study looks quite natural, and fits well with all the examples we have given. In the abelian cases where the partial multiplication is pointwise multiplication or convolution of functions, one even gets tight topological partial *-algebras. In the more interesting case of partial *-algebras of operators, the definition still works, but the validity of the tightness condition is generally open. It is satisfied for the 'nicest' infinite scale, namely that built on the powers of a selfadjoint operator, but it is not for a finite scale in general. In fact, it is not clear how much this condition is needed. It will obviously play a role in the definition of representations by the GNS construction [10]. When it is satisfied, it may offer interesting approximation procedures, following the standard pattern of functional analysis.

Of course, many open questions remain, in particular for partial $\mathrm{O}^{*}$-algebras. Another challenging problem is how to use this technique for extending representations of

*-algebras to partial *-algebras, for instance, a GNS representation. However, as we emphasized in the introduction, this paper is only a first step toward a general theory. Our aim was to find a structure suitable for as many significant examples as possible, and that has been obtained. But presumably the resulting framework is too general, and one ought to specialize it to particular cases. Clearly, more experience in this direction is needed before significant progress can be made.

\section{Appendix: Proof of Proposition 5.1}

\section{Acknowledgements}

This work was performed in the Institut de Physique Théorique, Université Catholique de Louvain, and the Istituto di Fisica dell' Università di Palermo. We thank both institutions for their hospitality, as well as financial support from CGRI, Communauté Française de Belgique, and Ministero degli Affari Esteri, Italy. 


\section{References}

[1] J-P. Antoine and A. Grossmann, Partial inner product spaces. I. General properties. II. Operators, J. Funct. Anal. 23 (1976) 369-378, 379-391

[2] J-P. Antoine, Partial inner product spaces. III. Compatibility relations revisited, J. Math. Phys. 21 (1980) 268-279

[3] J-P. Antoine, Partial inner product spaces. IV. Topological considerations, J. Math. Phys. 21 (1980) 2067-2079

[4] J-P. Antoine and K. Gustafson, Partial inner product spaces and semi-inner product spaces, Adv. in Math. 41 (1981) 281-300

[5] J-P. Antoine and W. Karwowski, Interpolation theory and refinement of nested Hilbert spaces, J. Math. Phys. 22 (1981) 2489-2496

[6] J-P. Antoine and W. Karwowski, Partial *-algebras of closed linear operators in Hilbert space, Publ. RIMS, Kyoto Univ. 21 (1985) 205-236; Add./Err. ibid. 22 (1986) $507-511$

[7] J-P. Antoine and F. Mathot, Partial *-algebras of closed operators and their commutants. I. General structure, Ann. Inst. H. Poincaré 46 (1987) 299-324

[8] J-P. Antoine, F. Mathot and C. Trapani, Partial *-algebras of closed operators and their commutants. II. Commutants and bicommutants, Ann. Inst. H. Poincaré 46 (1987) 325-351

[9] J-P. Antoine, A. Inoue and C. Trapani, Partial *-algebras of closable operators. I. The basic theory and the abelian case, Publ. RIMS, Kyoto Univ. 26 (1990) 359-395

[10] J-P. Antoine, A. Inoue and C. Trapani, Partial *-algebras of closable operators. II. States and representations of partial *-algebras, Publ. RIMS, Kyoto Univ. 27 (1991) 399-430

[11] J-P. Antoine, A. Inoue and C. Trapani, On the regularity of partial $\mathrm{O}^{*}$-algebras of generated by a closed symmetric operator, Publ. RIMS, Kyoto Univ. 28 (1992) $757-774$

[12] J-P. Antoine, A. Inoue and C. Trapani, Partial *-algebras of closable operators: A review, Reviews Math. Phys. 8 (1996) 1-42 
[13] J-P. Antoine, Quantum mechanics beyond Hilbert space. Applications to scattering theory, in Quantum Theory in Rigged Hilbert Spaces - Semigroups, Irreversibilty and Causality, A. Böhm, H.D. Doebner and P. Kielanowski (eds.), Springer, Berlin, 1997 (to appear)

[14] J-P. Antoine, F. Bagarello and C. Trapani, Extension of representations in quasi*algebras, Ann. Inst. H. Poincaré (1998) (to appear)

[15] F. Bagarello and C. Trapani, States and representations of CQ*-algebras, Ann. Inst. H. Poincaré 61 (1994) 103-133

[16] F. Bagarello and C. Trapani, CQ*-algebras: Structure properties, Publ. RIMS, Kyoto Univ. 32 (1996) 85-116

[17] F. Bagarello and C. Trapani, The Heisenberg dynamics of spin systems: A quasi *-algebras approach, J. Math. Phys. 37 (1996) 4219-4234

[18] F. Bagarello, A. Inoue and C. Trapani, Standard CQ*-algebras (in preparation)

[19] F. Bagarello and C. Trapani, $L^{p}$ spaces as quasi *-algebras, J. Math. Anal. Appl. 197 (1996) 810-824

[20] F. Bagarello and C. Trapani, CQ*-algebras of operators in scales of Hilbert spaces (in preparation)

[21] J.J. Benedetto, C. Heil and D.F. Walnut, Differentiation and the Balian-Low theorem, J. Fourier Anal. Appl. 1 (1995) 355-402

[22] J. Bergh and J. Löfström, Interpolation Spaces, Springer, Berlin, 1976

[23] H.J. Borchers, Decomposition of families of unbounded operators, in RCP 25 (Strasbourg) 22 (1975) 26-53; also in Quantum Dynamics: Models and Mathematics (L.Streit,ed.), Acta Phys. Austr. Suppl. 16 (1976) 15

[24] H.W. Davis, F.J. Murray and J.K. Weber, Families of $L_{p}$ spaces with inductive and projective topologies, Pacific J. Math. 34 (1970) 619-638; Inductive and projective limits of $L_{p}$ spaces, Portug. Math. 31 (1972) 21-29

[25] J.J.F. Fournier and J. Stewart, Amalgams of $L^{p}$ and $\ell^{q}$, Bull. Amer. Math. Soc. 13 (1985) 1-21

[26] F. Holland, Harmonic analysis on amalgams of $L^{p}$ and $\ell^{q}$, J. London Math. Soc. (2) 10 (1975) 295-305 
[27] K-D. Kürsten, The completion of the maximal Op*-algebra on a Fréchet domain, Publ. RIMS, Kyoto Univ. 22 (1986) 151-175; On topological linear spaces of operators with a unitary domain of definition, Wiss. Z. Univ-Leipzig, Math.-Naturwiss. R. 39 (1990) 623-655

[28] G. Lassner, Quasi-uniform topologies on local observables, Acta Univ. Wrat. No. 519 (Proc. Karpacz 1979), pp. 44-60; Wroclaw, 1979; $\beta$-topologies on operator algebras, in Coll. Int. CNRS No.274, "Algèbres d'opérateurs et leurs applications en physique mathématique" (Marseille 197r), pp. .....

[29] G. Lassner, Topological algebras and their applications in Quantum Statistics, Wiss. Z. KMU-Leipzig, Math.-Naturwiss. R. 30 (1981) 572-595

[30] G. Lassner, Algebras of unbounded operators and quantum dynamics, Physica 124 A (1984) 471-480

[31] F. Mathot, Topological properties of unbounded bicommutants, J. Math. Phys. 26 (1985) 1118-1124

[32] A. Russo and C. Trapani, Quasi *-algebras and multiplication of distributions, J. Math. Anal. Appl. (1997) (to appear)

[33] H.H. Schaefer, Topological Vector Spaces, Springer-Verlag, Berlin, 1971

[34] K. Schmüdgen, Unbounded Operator Algebras and Representation Theory, AkademieVerlag, Berlin, 1990

[35] B. Simon, The Hermite representation of tempered distributions (???), J. Math. Phys. .. (19..) ...

[36] S. Stratila and L. Zsido, Lectures on Von Neumann algebras, Abacus Press, Tunbridge Wells (England), 1979

[37] C. Trapani, Quasi *-algebras of operators and their applications, Reviews Math. Phys. 7 (1995) 1303-1332

[38] N. Wiener, On the representation of functions by trigonometric integrals, Math. Z. 24 (1926) 575-616; Tauberian theorems, Annals of Math. 33 (1932) 1-100

[39] A.C. Zaanen, Integration, 2nd. ed., Chap. 15; North-Holland, Amsterdam, 1961 